\title{
Coordination of thrusters, reaction wheels, and arm in orbital robots
}

\author{
Alessandro M. Giordano a,b,*, Alexander Dietrich ${ }^{\text {a }}$, Christian Ott ${ }^{\text {, }}$, Alin Albu-Schäffer a,b \\ ${ }^{a}$ Institute of Robotics and Mechatronics, German Aerospace Center (DLR), 82234 Weßling, Germany \\ b Department of Informatics, Technische Universität München (TUM), Garching, 85748, Germany
}

\section{A R T I C L E I N F O}

\section{Article history:}

Received 9 August 2019

Received in revised form 7 May 2020

Accepted 13 May 2020

Available online 20 May 2020

\section{Keywords:}

Space robotics

Thrusters

Reaction wheels

Stability analysis

Nonlinear control

Hardware-in-the-loop

\begin{abstract}
A B S T R A C T
A fuel-efficient control strategy for a manipulator-equipped spacecraft is presented. The strategy uses the thrusters, the reaction wheels, and the arm drives in a coordinated way to limit the use of the thrusters and achieve ideally zero fuel consumption in contact-free maneuvering. The thrusters are activated automatically only after contact, to stabilize the inertial motion of the system. The controller regulates the translation of the center-of-mass (CoM) of the whole space robot, the rotation of the spacecraft, and the pose of the end-effector (EE) in a decoupled way, utilizing the thrusters to control the CoM translation only and the remaining actuators to control the rotation and endeffector coordinately. The method is validated experimentally using a hardware-in-the-loop simulator composed of a seven degrees-of-freedom (DOF) arm mounted on a 6DOF simulated spacecraft. Numerical simulations with discrete thrusters assess the fuel efficiency of the proposed strategy.
\end{abstract}

(C) 2020 Elsevier B.V. All rights reserved.

\section{Introduction}

Space robots represent a promising technology for on-orbit servicing and assembly. They can be applied to refuel, repair, and upgrade existing satellites [1, §55.1], to deorbit space debris [2], or to build large telescopes or space stations. In the past thirty years, the investigation of such complex systems led to intense theoretical and experimental research [3], as well as to the first in-orbit technology demonstrators. The task of grasping a cooperative target satellite with a space robot was performed in the Engineering Test Satellite VII (ETS-VII) and Orbital Express missions [1, §55.1]. The ETS-VII mission [4,5] was operated in space from 1997 to 1999, and demonstrated for the first time the successful approach and docking with a target object in orbit by means of a robotic arm.

The design of controllers for orbital robotic systems presents a number of challenges from the point of view of dynamics, sensing, and actuation. Some of the major challenges are the dynamic interaction between the spacecraft and the arm, which needs to be taken into account in order to ensure stability and achieve high-performance, and the heterogeneity of the actuators available for control, each one presenting different advantages and limitations. The drives of the arm and the momentum exchange devices can provide internal torque at high rates [2], but they cannot provide external forces and torques to control the inertial motion of the robot. Furthermore, the drives of the arm

\footnotetext{
* Corresponding author at: Department of Informatics, Technische Universität München (TUM), Garching, 85748, Germany.

E-mail address: alessandro.giordano@dlr.de (A.M. Giordano).
}

are normally capable of providing a much higher torque than the momentum exchange devices. On the other hand, thrusters can be commanded only in discrete on-off mode and at low control rate [2], but they are capable of providing external force and torques. Furthermore, thrusters consume precious nonrenewable fuel, reducing the useful life of the space robot in orbit, while the drives of the arm and the momentum exchange devices use electrical power, which is renewable in orbit thanks to the solar arrays of the spacecraft.

In the early control concepts, attention was given to the possibility of completely turning off the spacecraft's actuators, resulting in a system for which the arm is commanded to realize an end-effector task while the spacecraft is left free-floating [610]. Within this concept, a kinematics-based control was then presented in [7] based on the use of the so-called generalized Jacobian matrix. The dynamics problem was treated in [8], where a transposed Jacobian approach was used to design the end effector control, and the stability of the method was addressed. The analysis of redundant free-floating robots was studied first in [11,12], and in more recent works [13-15]. The trajectory planning problem was analyzed in [16-18]. The transposed-Jacobian control of a free-floating robot in presence on nonzero initial momenta was treated in [15]. The impedance control for a freefloating robot was presented in $[19,20]$, based on a feedback linearization approach. The problems of the contact dynamics and the impedance matching were discussed in [21-23].

Recently, it was observed how a pure free-floating strategy might have limited applicability [19,24]. Firstly, the drift induced on a free-floating robot by a single accidental or voluntary contact can lead to singularity of the arm within short time, as 
observed via numerical simulation in [19], and experimentally in [24]. In such cases, the use of the thrusters to at least stabilize the translational motion is unavoidable. Secondly, attitude pointing might be required by missions relying on a spacecraftmounted relative sensor, e.g. the e.deorbit mission of the European Space Agency [2]. In such cases, thrusters or momentum exchange devices may be required to control also the rotation of the spacecraft.

Thrusters-only coordinated control strategies were proposed in the literature to simultaneously control the arm, the attitude of the spacecraft, and to stabilize the translational motion [2530]. However, when momentum exchange devices, e.g. reaction wheels (RWs), are available on board, methods relying exclusively on thrusters may cause an unnecessary high fuel consumption and may be affected by other limitations, such as discretization [29]; a better solution would be to also make use of reaction wheels in the controller algorithm. Combining thrusters and reaction wheels may allow achieving the requirements with a reduced fuel consumption and increased performance. Although the coordination of thrusters and momentum exchange devices is not new in the field of single-body space systems, to the best of the authors' knowledge there is a substantial lack of methods to coordinately steer the thrusters, the momentum exchange devices, and the arm in case of a multibody system. A control strategy was derived in [31], which uses the thrusters for controlling the translation of the spacecraft and the momentum exchange devices for controlling the rotation, while the arm is commanded to follow a desired trajectory. However, the requirement of controlling the translation of the spacecraft leads to increased fuel consumption during the pre-contact phase of the robotic operation. A different and more fuel-efficient requirement of controlling the translation of the center-of-mass (CoM) of the whole space robot may be enforced for stabilizing the translational motion, as observed in [30,32]. Compared to a base-control strategy such as [31], a CoM-control approach employs ideally ${ }^{1}$ no thrusters during the pre-contact phase, because the CoM naturally conserves. Compared to a free-floating strategy, a CoM-control approach can stop the inertial drift during the post-contact phase. Compared to a switching strategy that employs free-floating control during the pre-contact phase and base control during the post-contact phase, a CoM-control approach allows using a single continuous controller and thus bypassing the main limitations of a switching approach. These are, for example, the requirement of contact detection for defining the switching condition, the possible occurrence of chattering or frequent switching due to measurements' uncertainties and/or nonuniform contact, and the necessity of a hybrid continuous-discontinuous stability proof of the ideal closed-loop system.

In this work, a control method is developed to regulate the CoM and the angular momentum of the whole space robot, the attitude of the spacecraft, and the pose of the end-effector; the tasks are achieved by a suitable coordinated actuation of the thrusters, the reaction wheels, and the arm. For this purpose, the method implements a dynamics transformation that features a total decoupling of the internal motion of both the reaction wheels and the arm from the external motion, and an inertial decoupling of the internal RWs and arm motion. Furthermore, the transformation features a decoupled allocation structure that assigns the end-effector and attitude control inputs only to the reaction wheels' and the joints' actuators, and not to the thrusters. To stabilize the translational motion, the method enforces a control of the translation of the CoM of the whole space robot

\footnotetext{
1 In other words, considering only the multi-body body behavior of the system and no uncertainties on thrusters and measurements.
}

(previously introduced in [32]) instead of a control of the translation of the spacecraft. Thanks to the dynamics transformation and to the control of the CoM, ideally zero fuel consumption during contact-free maneuvering is achieved, and the full stabilization of the system in the post-contact phase is still ensured. The contributions of the paper are:

- the transformation of the dynamics;

- the development of a coordinated control of the attitude of the spacecraft, the position of the CoM of the whole space robot, and the pose of the end-effector, which achieves ideally zero fuel consumption in pre-contact operations;

- the proof of stability;

- an experimental validation of the approach using a full 3D hardware-in-the-loop simulator (see Fig. 1).

Note that the transformation of the dynamics is different than the one derived in [30] and [32] because in [30] the attitude and end-effector control inputs are assigned to the thrusters torques; furthermore, [30] results in an inertially-coupled attitude and end-effector dynamics. In [32] the attitude cannot be controlled.

The paper is structured as follows: Section 2 introduces the main equations. Section 3 describes the decoupling of the dynamics. Section 4 presents the proposed controller and the stability analysis. Section 5 presents the experimental results. Section 6 analyzes the effects of real thrusters. Finally, concluding remarks are drawn.

\section{Preliminaries}

\subsection{Problem statement}

A space robot is considered, which is composed of a spacecraft, a serial-chain manipulator with $n_{m}$ revolute joints, and a system of $n_{w}$ reaction wheels with fixed gymbal.

The total number of degrees of freedom (DOFs) of the moving bodies is $6+n$, where $n=n_{w}+n_{m}$ is the total number of robot joints. The spacecraft is equipped with a set of $n_{t}$ thrusters, which exert forces and torques. The operational scenario involves the maneuvering of the space robot in the proximity of a target object (see Fig. 2) that is stationary in the inertial space. This may include the tasks of inspecting, capturing, or servicing a nontumbling object in orbit. No orbital or environmental forces are considered, because they are in practice considerably smaller than the control ones [2].

The problem addressed is the efficient coordination of joint drives, reaction wheels, and thrusters, so as to simultaneously regulate the pose of the end-effector, the attitude of the spacecraft, and the CoM and the angular momentum of the whole space robot. At this purpose, we seek at a controller satisfying the following two fundamental properties:

1. maneuvers of the arm and of the attitude of the spacecraft shall be entirely accomplished by the electrical drives of the reaction wheels and the arm, and not by the thrusters;

2. the thrusters shall only stabilize the angular momentum of the system and regulate the position of CoM of the whole space robot.

\subsection{Notation}

Fig. 2 shows a schematic representation of the system components. The following frames are considered: a base frame $\mathcal{B}$ attached to the spacecraft, an end-effector frame $\mathcal{E}$, a frame $\mathcal{T}$ attached to a target object, and a frame $\mathcal{C}$ located at the CoM of the whole space robot with axes fixed w.r.t. the inertial space. The joints of the reaction wheels are denoted by $\boldsymbol{q}_{w} \in \mathbb{R}^{n_{w}}$, the 


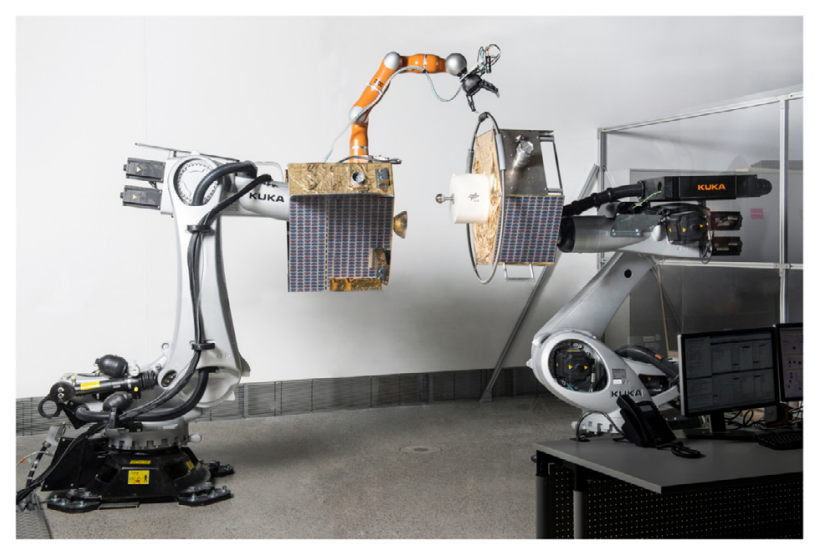

Fig. 1. The On-Orbit Servicing Simulator at DLR RMC: detail of the micro-macro space robot simulator.

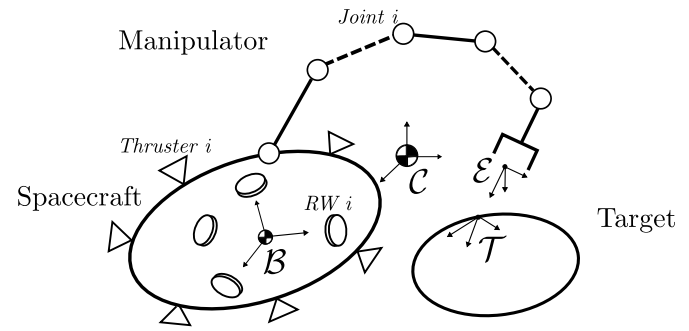

Fig. 2. Schematic representation of components of the space robot: a manipulator mounted on a thrusters- and reaction wheels-equipped spacecraft.

joints of the arm are denoted by $\boldsymbol{q}_{m} \in \mathbb{R}^{n_{m}}$. The quantities $\boldsymbol{f}_{b} \in \mathbb{R}^{3}$ and $\boldsymbol{\tau}_{b} \in \mathbb{R}^{3}$ denote the base force and torque about $\mathcal{B}$ acted upon by the thrusters and expressed in frame $\mathcal{B}$. Let us denote $\boldsymbol{p}_{x y} \in \mathbb{R}^{3}$ and $\boldsymbol{R}_{x y} \in \mathrm{SO}(3)$, the position vector from a general frame $\mathcal{X}$ to a general frame $\mathcal{Y}$ expressed in frame $\mathcal{X}$, and the corresponding rotation matrix, respectively. The so-called Adjoint transformation is used:

$\boldsymbol{A}_{x y}=\left[\begin{array}{cc}\boldsymbol{R}_{x y} & {\left[\boldsymbol{p}_{x y}\right]^{\wedge} \boldsymbol{R}_{x y}} \\ \mathbf{0} & \boldsymbol{R}_{x y}\end{array}\right] \in \mathbb{R}^{6 \times 6}$,

wherein the operator $[\cdot]^{\wedge}$ indicates the skew-symmetric matrix of the vector argument. Let us generally denote $\boldsymbol{v}_{x} \in \mathbb{R}^{3}$ and $\omega_{x} \in \mathbb{R}^{3}$ as the linear and angular velocities of a frame $\mathcal{X}$ relative to the inertial frame $\mathcal{T}$ expressed in $\mathcal{X}$, respectively. The symbol $\boldsymbol{v}_{x}=\left[\begin{array}{ll}\boldsymbol{v}_{x}^{T} & \boldsymbol{\omega}_{x}^{T}\end{array}\right]^{T} \in \mathbb{R}^{6}$ indicates the corresponding 6DOF velocity. The symbols $\mathbf{0}$ and $\boldsymbol{E}$ indicate the zero and identity matrices of suitable dimensions. The notation $\operatorname{diag}(a, b, c)$ indicates the diagonal matrix obtained from the scalars $a, b, c$, whereby blkdiag $(\boldsymbol{A}, \boldsymbol{B})$ indicates the block-diagonal matrix obtained from the matrices $\boldsymbol{A}$ and $\boldsymbol{B}$.

\subsection{Kinematics and dynamics}

The end-effector body velocity is given by

$\boldsymbol{v}_{e}=\boldsymbol{A}_{e b}\left(\boldsymbol{q}_{m}\right) \boldsymbol{v}_{b}+\boldsymbol{J}_{e m}\left(\boldsymbol{q}_{m}\right) \dot{\boldsymbol{q}}_{m}$,

where $\boldsymbol{J}_{e m}\left(\boldsymbol{q}_{m}\right) \in \mathbb{R}^{6 \times n_{m}}$ is the manipulator Jacobian matrix. For the derivation of the dynamics model the following assumption is made:

Assumption 1. Each reaction wheel is a rotational symmetric rigid body, whose rotation and symmetry axes coincide.
The dynamics of the space robot in absence of contact is computed [33] as

$$
\begin{aligned}
\underbrace{\left[\begin{array}{ccc}
\boldsymbol{M}_{b} & \boldsymbol{M}_{b w} & \boldsymbol{M}_{b m} \\
\boldsymbol{M}_{b w}^{T} & \boldsymbol{M}_{w} & \mathbf{0} \\
\boldsymbol{M}_{b m}^{T} & \mathbf{0} & \boldsymbol{M}_{m}
\end{array}\right]}_{\boldsymbol{M}\left(\boldsymbol{q}_{m}\right)}\left[\begin{array}{c}
\dot{\boldsymbol{v}}_{b} \\
\ddot{\boldsymbol{q}}_{w} \\
\ddot{\boldsymbol{q}}_{m}
\end{array}\right] \\
+\underbrace{\left[\begin{array}{ccc}
\boldsymbol{C}_{b} & \boldsymbol{C}_{b w} & \boldsymbol{C}_{b m} \\
\boldsymbol{C}_{w b} & \boldsymbol{C}_{w} & \mathbf{0} \\
\boldsymbol{C}_{m b} & \mathbf{0} & \boldsymbol{C}_{m}
\end{array}\right]}_{\boldsymbol{C}\left(\boldsymbol{q}_{m}, \boldsymbol{v}_{b}, \dot{\boldsymbol{q}}_{w}, \dot{\boldsymbol{q}}_{m}\right)} \underbrace{\left[\begin{array}{c}
\boldsymbol{v}_{b} \\
\dot{\boldsymbol{q}}_{w} \\
\dot{\boldsymbol{q}}_{m}
\end{array}\right]}_{\boldsymbol{v}}=\underbrace{\left[\begin{array}{c}
\mathcal{F}_{b} \\
\boldsymbol{\tau}_{w} \\
\boldsymbol{\tau}_{m}
\end{array}\right]}_{\boldsymbol{f}},
\end{aligned}
$$

where $\boldsymbol{v} \in \mathbb{R}^{6+n}$ and $\boldsymbol{f} \in \mathbb{R}^{6+n} ; \quad \boldsymbol{M}\left(\boldsymbol{q}_{m}\right) \in \mathbb{R}^{(6+n) \times(6+n)}$ and $\boldsymbol{C}\left(\boldsymbol{q}_{m}, \boldsymbol{v}_{b}, \dot{\boldsymbol{q}}_{w}, \dot{\boldsymbol{q}}_{m}\right) \in \mathbb{R}^{(6+n) \times(6+n)}$ are the inertia and Coriolis/ centrifugal matrices, respectively; $\mathcal{F}_{b}=\left[\begin{array}{cc}\boldsymbol{f}_{b}^{T} & \boldsymbol{\tau}_{b}^{T}\end{array}\right]^{T} \in \mathbb{R}^{6}$ is the wrench of the thrusters at the base; $\boldsymbol{\tau}_{w} \in \mathbb{R}^{n_{w}}$ and $\boldsymbol{\tau}_{m} \in \mathbb{R}^{n_{m}}$ are the wheel and joint torques, respectively. The expression of the dynamic matrices is reported in Appendix A. From now on, the functional dependency of the matrices is omitted for the sake of readability; it will be recovered only when explicitly needed. Denoting $\boldsymbol{p} \in \mathbb{R}^{3}$ the linear momentum, and $\boldsymbol{l} \in \mathbb{R}^{3}$ the angular momentum around $\mathcal{C}$, the generalized momentum $\boldsymbol{h}=\left[\begin{array}{ll}\boldsymbol{p}^{T} & \boldsymbol{l}^{T}\end{array}\right]^{T} \in \mathbb{R}^{6}$ is given by

$\boldsymbol{h}=\boldsymbol{A}_{c b}^{-T}\left(\boldsymbol{M}_{b} \boldsymbol{v}_{b}+\boldsymbol{M}_{b w} \dot{\boldsymbol{q}}_{w}+\boldsymbol{M}_{b m} \dot{\mathbf{q}}_{m}\right)$.

The velocity of the CoM $\boldsymbol{v}_{c} \in \mathbb{R}^{3}$ is obtained as

$\boldsymbol{v}_{c}=\frac{1}{m} \boldsymbol{p}=\frac{1}{m} \boldsymbol{Q}_{c} \boldsymbol{h}$,

where $\boldsymbol{Q}_{c}=\left[\begin{array}{ll}\boldsymbol{E} & \mathbf{0}\end{array}\right] \in \mathbb{R}^{3 \times 6}$. In the next section, the transformation of the dynamics into an advantageous decoupled form is addressed.

\section{Decoupled dynamics}

\subsection{Internal velocity}

In [24], the motion of the end-effector frame was decomposed into an internal one due to the joints and an external one as a whole. This was achieved by application of the concept of internal velocity, which allows the decoupling of the internal end-effector dynamics from the momentum dynamics. The same concept is stated here for general frames and for the case in which reaction wheels are present in the system.

The "external motion" is defined as the part of the motion related to the kinetic momentum of the system. In turn, the internal motion $v_{x}^{\text {int }} \in \mathbb{R}^{6}$ of the general frame $\mathcal{X}$ is defined as its motion $v_{x}$ when the external motion is zero; in other words, when the momentum is zero, $\boldsymbol{v}_{x}$ must coincide with $\boldsymbol{v}_{x}^{\text {int }}$ :

$\boldsymbol{h}=\mathbf{0} \Longleftrightarrow \boldsymbol{v}_{x}=\boldsymbol{v}_{x}^{\text {int }}$.

Let us consider the end-effector frame. Its velocity can be written by eliminating $\boldsymbol{v}_{b}$ from (4) and (2), as

$\boldsymbol{v}_{e}=\boldsymbol{A}_{e b} \boldsymbol{M}_{b}^{-1} \boldsymbol{A}_{c b}^{T} \boldsymbol{h}-\boldsymbol{A}_{e b} \boldsymbol{M}_{b}^{-1} \boldsymbol{M}_{b w} \dot{\boldsymbol{q}}_{w}+\boldsymbol{J}_{e m}^{*} \dot{\boldsymbol{q}}_{m}$,

where $\boldsymbol{J}_{e m}^{*}=\boldsymbol{J}_{e m}-\boldsymbol{A}_{e b} \boldsymbol{M}_{b}^{-1} \boldsymbol{M}_{b m} \in \mathbb{R}^{6 \times n_{m}}$ is the so-called generalized Jacobian matrix $[7,8]$ of the end-effector. Then, by setting $\boldsymbol{h}=\mathbf{0}$ in (7), it is clearly seen that the end-effector internal velocity is

$\boldsymbol{v}_{e}^{i n t}=-\boldsymbol{A}_{e b} \boldsymbol{M}_{b}^{-1} \boldsymbol{M}_{b w} \dot{\boldsymbol{q}}_{w}+\boldsymbol{J}_{e m}^{*} \dot{\boldsymbol{q}}_{m}$,

and that (7) can be rewritten as

$\boldsymbol{v}_{e}=\boldsymbol{A}_{e b} \boldsymbol{M}_{b}^{-1} \boldsymbol{A}_{c b}^{T} \boldsymbol{h}+\boldsymbol{v}_{e}^{i n t}$. 
Notice that in the presence of reaction wheels, the end-effector internal velocity (8a) is not a function of the joint velocities of the arm only, as in [24], but it also depends on the velocities of the wheels. This is explained by the fact that the reaction wheels are dynamically coupled with the base, which is in turn dynamically coupled with the arm, resulting in an indirect coupling of wheels and arm.

Considering the base frame, by similarly setting $\boldsymbol{h}=\mathbf{0}$ in (4), the base internal velocity is given by

$\boldsymbol{v}_{b}^{i n t}=-\boldsymbol{M}_{b}^{-1} \boldsymbol{M}_{b w} \dot{\boldsymbol{q}}_{w}-\boldsymbol{M}_{b}^{-1} \boldsymbol{M}_{b m} \dot{\boldsymbol{q}}_{m}$,

and (4) can be rewritten as

$\boldsymbol{v}_{b}=\boldsymbol{M}_{b}^{-1} \boldsymbol{A}_{c b}^{T} \boldsymbol{h}+\boldsymbol{v}_{b}^{\text {int }}$.

In this work, the base translation is not controlled, so let us consider only the angular part of (9), which is given by:

$\boldsymbol{\omega}_{b}^{i n t}=-\boldsymbol{Q}_{b} \boldsymbol{M}_{b}^{-1} \boldsymbol{M}_{b w} \dot{\boldsymbol{q}}_{w}-\boldsymbol{Q}_{b} \boldsymbol{M}_{b}^{-1} \boldsymbol{M}_{b m} \dot{\boldsymbol{q}}_{m}$,

$\boldsymbol{\omega}_{b}=\boldsymbol{Q}_{b} \boldsymbol{M}_{b}^{-1} \boldsymbol{A}_{c b}^{T} \boldsymbol{h}+\boldsymbol{\omega}_{b}^{\text {int }}$,

where $\boldsymbol{Q}_{b}=\left[\begin{array}{ll}\mathbf{0} & \boldsymbol{E}\end{array}\right] \in \mathbb{R}^{3 \times 6}$.

For later reference, let us compactly write (8a), (10a), as

$\boldsymbol{v}_{e}^{\text {int }}=\left[\begin{array}{ll}\boldsymbol{T}_{e w} & \boldsymbol{T}_{e m}\end{array}\right] \dot{\boldsymbol{q}}=\boldsymbol{T}_{e} \dot{\boldsymbol{q}}$,

$\boldsymbol{\omega}_{b}^{\text {int }}=\left[\begin{array}{ll}\boldsymbol{T}_{b w} & \boldsymbol{T}_{b m}\end{array}\right] \dot{\boldsymbol{q}}=\boldsymbol{T}_{b} \dot{\boldsymbol{q}}$,

where $\boldsymbol{T}_{e} \in \mathbb{R}^{6 \times n}, \boldsymbol{T}_{b} \in \mathbb{R}^{3 \times n}$, and $\dot{\boldsymbol{q}}=\left[\begin{array}{ll}\dot{\boldsymbol{q}}_{w}^{T} & \dot{\boldsymbol{q}}_{m}^{T}\end{array}\right]^{T} \in \mathbb{R}^{n}$. The submatrices are $\boldsymbol{T}_{e w}=-\boldsymbol{A}_{e b} \boldsymbol{M}_{b}^{-1} \boldsymbol{M}_{b w} \in \mathbb{R}^{6 \times n_{w}}, \boldsymbol{T}_{e m}=\boldsymbol{J}_{e m}^{*} \in \mathbb{R}^{6 \times n_{m}}$, $\boldsymbol{T}_{b w}=-\boldsymbol{Q}_{b} \boldsymbol{M}_{b}^{-1} \boldsymbol{M}_{b w} \in \mathbb{R}^{3 \times n_{w}}$, and $\boldsymbol{T}_{b m}=-\boldsymbol{Q}_{b} \boldsymbol{M}_{b}^{-1} \boldsymbol{M}_{b m} \in \mathbb{R}^{3 \times n_{m}}$.

The advantage of the velocities $\boldsymbol{v}_{e}^{\text {int }}$ and $\boldsymbol{\omega}_{b}^{\text {int }}$ in comparison to $v_{e}$ and $\omega_{b}$, is that they allow transforming the dynamics in a decoupled form in which end-effector and attitude control inputs are not actuated by thrusters, but only by internal actuators. This will be demonstrated in the next section.

\subsection{External-internal decomposition}

Based on (4), (8a) and (10a), the following transformation $\Gamma \in$ $\mathbb{R}^{15 \times(6+n)}$ is defined:

$\left[\begin{array}{c}\boldsymbol{h} \\ \boldsymbol{v}_{e}^{\text {int }} \\ \boldsymbol{\omega}_{b}^{\text {int }}\end{array}\right]=\underbrace{\left[\begin{array}{ccc}\boldsymbol{A}_{c b}^{-T} \boldsymbol{M}_{b} & \boldsymbol{A}_{c b}^{-T} \boldsymbol{M}_{b w} & \boldsymbol{A}_{c b}^{-T} \boldsymbol{M}_{b m} \\ \mathbf{0} & \boldsymbol{T}_{e w} & \boldsymbol{T}_{e m} \\ \mathbf{0} & \boldsymbol{T}_{b w} & \boldsymbol{T}_{b m}\end{array}\right]}_{\boldsymbol{\Gamma}}\left[\begin{array}{c}\boldsymbol{v}_{b} \\ \dot{\boldsymbol{q}}_{w} \\ \dot{\boldsymbol{q}}_{m}\end{array}\right]$,

The generalized forces transform through

$\left[\begin{array}{c}\mathcal{F}_{b} \\ \boldsymbol{\tau}_{w} \\ \boldsymbol{\tau}_{m}\end{array}\right]=\Gamma^{T}\left[\begin{array}{c}\boldsymbol{a}_{c} \\ \mathcal{F}_{e}^{i n t} \\ \boldsymbol{\tau}_{b}^{i n t}\end{array}\right]$,

with $\boldsymbol{a}_{c} \in \mathbb{R}^{6}, \mathcal{F}_{e}^{\text {int }} \in \mathbb{R}^{6}$ and $\boldsymbol{\tau}_{b}^{\text {int }} \in \mathbb{R}^{3}$ being new control inputs: $\boldsymbol{a}_{c}$ is the virtual centroidal acceleration , $\mathcal{F}_{e}^{\text {int }}$ is a virtual end-effector wrench, and $\boldsymbol{\tau}_{b}^{\text {int }}$ is a virtual base torque.

For the derivation of the dynamics in the transformed space, the following two assumptions are made:

Assumption 2. The set of reaction wheels is nonredundant, i.e., $n_{w}=3$, and nonsingular.

Assumption 3. The arm is nonredundant, i.e., $n_{m}=6$.

Resolution methods for redundant kinematic chains are available in the literature of floating robots [13-15] which might be applied to the present case. However, the extension of the method to redundant arms is omitted herein for brevity and will be subject of future work.
The mapping $\boldsymbol{\Gamma}\left(\boldsymbol{R}_{b}, \boldsymbol{q}_{m}\right)$ is not rank constant because is function of $\boldsymbol{R}_{b}$ and $\boldsymbol{q}_{m}$. Based on the triangular form (12), and considering that $\boldsymbol{A}_{c b}\left(\boldsymbol{R}_{b}, \boldsymbol{q}_{m}\right)^{-T} \boldsymbol{M}_{b}\left(\boldsymbol{q}_{m}\right)$ is always full rank, $\boldsymbol{\Gamma}\left(\boldsymbol{R}_{b}, \boldsymbol{q}_{m}\right)$ is singular when

$\boldsymbol{T}\left(\boldsymbol{q}_{m}\right)=\left[\begin{array}{l}\boldsymbol{T}_{e}\left(\boldsymbol{q}_{m}\right) \\ \boldsymbol{T}_{b}\left(\boldsymbol{q}_{m}\right)\end{array}\right]$

is singular, meaning also that the singularity of $\Gamma\left(\boldsymbol{R}_{b}, \boldsymbol{q}_{m}\right)$ depends only on the joints. Thus, when $\boldsymbol{T}\left(\boldsymbol{q}_{m}\right)$ is nonsingular, the inverse of $\boldsymbol{\Gamma}\left(\boldsymbol{R}_{b}, \boldsymbol{q}_{m}\right)$ is well defined, and is used to transform (3) as [34, p. 32]:

$$
\begin{aligned}
{\left[\begin{array}{ccc}
\boldsymbol{M}_{c}^{-1} & \mathbf{0} & \mathbf{0} \\
\mathbf{0} & \overline{\boldsymbol{M}}_{e} & \overline{\boldsymbol{M}}_{e b} \\
\mathbf{0} & \overline{\boldsymbol{M}}_{e b}^{T} & \overline{\boldsymbol{M}}_{b}
\end{array}\right]\left[\begin{array}{c}
\dot{\boldsymbol{h}}^{i n t} \\
\dot{\boldsymbol{v}}_{e}^{\text {int }} \\
\dot{\boldsymbol{\omega}}_{b}^{\text {int }}
\end{array}\right] } \\
+\left[\begin{array}{ccc}
\boldsymbol{C}_{h} & -\overline{\boldsymbol{C}}_{e h}^{T} & -\overline{\boldsymbol{C}}_{b h}^{T} \\
\overline{\boldsymbol{C}}_{e h} & \overline{\boldsymbol{C}}_{e} & \overline{\boldsymbol{C}}_{e b} \\
\overline{\boldsymbol{C}}_{b h} & \overline{\boldsymbol{C}}_{b e} & \overline{\boldsymbol{C}}_{b}
\end{array}\right]\left[\begin{array}{c}
\boldsymbol{h} \\
\boldsymbol{v}_{e}^{i n t} \\
\boldsymbol{\omega}_{b}^{\text {int }}
\end{array}\right]=\left[\begin{array}{c}
\boldsymbol{a}_{c} \\
\mathcal{F}_{e}^{\text {int }} \\
\boldsymbol{\tau}_{b}^{i n t}
\end{array}\right],
\end{aligned}
$$

where $\boldsymbol{M}_{c}=\boldsymbol{A}_{c b}^{-T} \boldsymbol{M}_{b} \boldsymbol{A}_{c b}^{-1} \in \mathbb{R}^{6}$ is the inertia of the whole system around $\mathcal{C}$ and $\overline{\boldsymbol{M}}=\left[\begin{array}{cc}\overline{\boldsymbol{M}}_{e} & \overline{\boldsymbol{M}}_{e b} \\ \overline{\boldsymbol{M}}_{e b} & \overline{\boldsymbol{M}}_{b}\end{array}\right] \in \mathbb{R}^{9 \times 9}$ is the inertia matrix associated to the end-effector and base system. Notice that the momentum is inertially decoupled from the rest of the system, as a consequence of the use of the internal velocities $v_{e}^{\text {int }}$ and $\omega_{b}^{\text {int }}$. Note that this advantageous decoupling does not hold when using $\boldsymbol{v}_{e}$ and $\boldsymbol{\omega}_{b}$ instead of $\boldsymbol{v}_{e}^{\text {int }}$ and $\boldsymbol{\omega}_{b}^{\text {int }}$. However, despite this advantage the internal dynamics in (14) is still fully coupled. In order to avoid interference of the base dynamics into the end-effector dynamics, it is convenient to adopt an alternative parametrization of the internal motion to achieve a complete decoupling. This is derived in the next section.

\subsection{External-internal decomposition with internal decoupling}

Considering Assumptions 2 and 3, it is $n=9$. For nonsingular configurations of $\boldsymbol{T}_{e}$, i.e., such that $\operatorname{rank}\left(\boldsymbol{T}_{e}\right)=6, \boldsymbol{T}_{e}$ possesses a null space of dimension 3 . Then, a new base velocity can be defined as the projection of the base internal angular velocity into the null space of the end-effector internal velocity, as

$\breve{\boldsymbol{\omega}}_{b}^{\text {int }}=\breve{\boldsymbol{T}}_{b} \dot{\boldsymbol{q}}, \quad$ with $\quad \breve{\boldsymbol{T}}_{b}=\boldsymbol{T}_{b} \boldsymbol{N}_{e}$,

and where

$\boldsymbol{N}_{e}^{T}=\boldsymbol{E}-\boldsymbol{T}_{e}^{T} \boldsymbol{T}_{e}^{\# T} \in \mathbb{R}^{n \times n}$

is the dynamically-consistent projector of $\omega_{b}^{\text {int }}$ onto the null space of the Jacobian matrix related to $\boldsymbol{v}_{e}^{\text {int }}$. Given a general matrix $\boldsymbol{P} \in$ $\mathbb{R}^{s \times p}$, the notation $\boldsymbol{P}^{\#} \in \mathbb{R}^{p \times s}$ indicates the dynamically-consistent pseudo-inverse [35] of $\boldsymbol{P}$, defined as

$\boldsymbol{P}^{\#}=\overline{\mathbf{M}}^{-1} \boldsymbol{P}^{T}\left(\boldsymbol{P} \overline{\mathbf{M}}^{-1} \boldsymbol{P}^{T}\right)^{-1}$.

Based on (15), a new set of internal velocities is defined as

$\left[\begin{array}{c}\boldsymbol{v}_{e}^{\text {int }} \\ \breve{\boldsymbol{\omega}}_{b}^{\text {int }}\end{array}\right]=\left[\begin{array}{c}\mathbf{T}_{e} \\ \breve{\boldsymbol{T}}_{b}\end{array}\right] \dot{\boldsymbol{q}}=\breve{\boldsymbol{T}} \dot{\mathbf{q}}$.

with $\breve{\boldsymbol{T}} \in \mathbb{R}^{9 \times n}$. In turn, let us define a new transformation $\breve{\Gamma} \in \mathbb{R}^{15 \times(6+n)}$, as

$\left[\begin{array}{c}\boldsymbol{h} \\ \boldsymbol{v}_{e}^{i n t} \\ \breve{\boldsymbol{\omega}}_{b}^{i n t}\end{array}\right]=\underbrace{\left[\begin{array}{ccc}\boldsymbol{A}_{c b}^{-T} \boldsymbol{M}_{b} & \boldsymbol{A}_{c b}^{-T} \boldsymbol{M}_{b w} & \boldsymbol{A}_{c b}^{-T} \boldsymbol{M}_{b m} \\ \mathbf{0} & \boldsymbol{T}_{e w} & \boldsymbol{T}_{e m} \\ \mathbf{0} & \breve{\boldsymbol{T}}_{b w} & \breve{\boldsymbol{T}}_{b m}\end{array}\right]}_{\breve{\boldsymbol{\Gamma}}}\left[\begin{array}{c}\boldsymbol{v}_{b} \\ \dot{\boldsymbol{q}}_{w} \\ \dot{\boldsymbol{q}}_{m}\end{array}\right]$, 
where $\breve{\boldsymbol{T}}_{b w} \in \mathbb{R}^{3 \times n_{w}}$ and $\breve{\boldsymbol{T}}_{b m} \in \mathbb{R}^{3 \times n_{m}}$ are the sub-matrices of the partitioning $\breve{\boldsymbol{T}}_{b}=\left[\begin{array}{cc}\breve{\boldsymbol{T}}_{b w} & \breve{\boldsymbol{T}}_{b m}\end{array}\right] \in \mathbb{R}^{3 \times n}$. The generalized forces transform as

$$
\left[\begin{array}{c}
\mathcal{F}_{b} \\
\boldsymbol{\tau}_{w} \\
\boldsymbol{\tau}_{m}
\end{array}\right]=\breve{\boldsymbol{\Gamma}}^{T}\left[\begin{array}{c}
\boldsymbol{a}_{c} \\
\breve{\mathcal{F}}_{e}^{i n t} \\
\breve{\boldsymbol{\tau}}_{b}^{i n t}
\end{array}\right]
$$

where $\breve{\mathcal{F}}_{e}^{\text {int }} \in \mathbb{R}^{6}$ and $\breve{\boldsymbol{\tau}}_{b}^{\text {int }} \in \mathbb{R}^{3}$ are new control inputs. By applying a similar reasoning as in Section 3.2, given the triangular form (19), $\breve{\boldsymbol{\Gamma}}\left(\boldsymbol{R}_{b}, \boldsymbol{q}_{m}\right)$ is singular when $\breve{\boldsymbol{T}}\left(\boldsymbol{q}_{m}\right)$ is singular. Thus, when $\breve{\boldsymbol{T}}\left(\boldsymbol{q}_{m}\right)$ is nonsingular, the inverse of $\breve{\boldsymbol{\Gamma}}\left(\boldsymbol{R}_{b}, \boldsymbol{q}_{m}\right)$ is well defined and is used to transform (3) to the form

$$
\begin{aligned}
\underbrace{\left[\begin{array}{ccc}
\boldsymbol{M}_{c}^{-1} & \mathbf{0} & \mathbf{0} \\
\mathbf{0} & \boldsymbol{M}_{e}^{*} & \mathbf{0} \\
\mathbf{0} & \mathbf{0} & \boldsymbol{M}_{b}^{*}
\end{array}\right]}_{\breve{\boldsymbol{M}}}\left[\begin{array}{c}
\dot{\boldsymbol{h}} \\
\dot{\boldsymbol{v}}_{e}^{i n t} \\
\dot{\boldsymbol{\omega}}_{b}^{i n t}
\end{array}\right] \\
+\underbrace{\left[\begin{array}{ccc}
\boldsymbol{C}_{h} & -\boldsymbol{C}_{e h}^{* T} & -\boldsymbol{C}_{b h}^{* T} \\
\boldsymbol{C}_{e h}^{*} & \boldsymbol{C}_{e}^{*} & -\boldsymbol{C}_{b e}^{* T} \\
\boldsymbol{C}_{b h}^{*} & \boldsymbol{C}_{b e}^{*} & \boldsymbol{C}_{b}^{*}
\end{array}\right]}_{\breve{\boldsymbol{C}}}\left[\begin{array}{c}
\boldsymbol{h} \\
\boldsymbol{v}_{e}^{i n t} \\
\breve{\boldsymbol{\omega}}_{b}^{i n t}
\end{array}\right]=\left[\begin{array}{c}
\boldsymbol{a}_{c} \\
\breve{\mathcal{F}}_{e}^{i n t} \\
\breve{\boldsymbol{\tau}}_{b}^{i n t}
\end{array}\right] .
\end{aligned}
$$

Notice that in (21) the system is completely block-decoupled in the inertial terms; namely, not only the momentum but also the internal end-effector and base inertial terms are now decoupled. The dynamics (21) can be further simplified by considering that the Coriolis and centrifugal vector terms are zero for the momentum equation (see Appendix A), i.e., $\boldsymbol{C}_{h} \boldsymbol{h}-\boldsymbol{C}_{e h}^{* T} \boldsymbol{v}_{e}^{\text {int }}-\boldsymbol{C}_{b h}^{* T} \breve{\boldsymbol{\omega}}_{b}^{\text {int }}=\mathbf{0}$. Considering this, and defining a centroidal control wrench $\mathcal{F}_{c} \in \mathbb{R}^{6}$ such that $\boldsymbol{a}_{c}=\boldsymbol{M}_{c}^{-1} \mathcal{F}_{c}$, the dynamics (21) simplifies to

$\dot{\boldsymbol{h}}=\mathcal{F}_{c}$,

$$
\left[\begin{array}{cc}
\boldsymbol{M}_{e}^{*} & \mathbf{0} \\
\mathbf{0} & \boldsymbol{M}_{b}^{*}
\end{array}\right]\left[\begin{array}{c}
\dot{\boldsymbol{v}}_{e}^{\text {int }} \\
\dot{\boldsymbol{\omega}}_{b}^{\text {int }}
\end{array}\right]+\left[\begin{array}{cc}
\boldsymbol{C}_{e}^{*} & -\boldsymbol{C}_{b e}^{* T} \\
\boldsymbol{C}_{b e}^{*} & \boldsymbol{C}_{b}^{*}
\end{array}\right]\left[\begin{array}{c}
\boldsymbol{v}_{e}^{i n t} \\
\breve{\boldsymbol{\omega}}_{b}^{\text {int }}
\end{array}\right]+\left[\begin{array}{c}
\boldsymbol{C}_{e h}^{*} \\
\boldsymbol{C}_{b h}^{*}
\end{array}\right] \boldsymbol{h}=\left[\begin{array}{c}
\breve{\mathcal{F}}_{e}^{i n t} \\
\breve{\boldsymbol{\tau}}_{b}^{\text {int }}
\end{array}\right]
$$

Notice that the form (22) is triangular, i.e., the external dynamics is completely decoupled from the internal one. This allows to straightforwardly design controllers for the external and internal motion in a cascaded fashion, as done in [24], i.e., designing the momentum control loop independently from the internal loop. It is relevant to note that the subblocks in (22) are not all equivalent to those associated to the end-effector and base motion in (14). In fact, it can be proven that $\boldsymbol{M}_{b}^{*}=\overline{\boldsymbol{M}}_{b}$ but $\boldsymbol{M}_{e}^{*} \neq \overline{\boldsymbol{M}}_{e}$. Lastly, it is remarked that the following properties hold for the matrices in (22b):

$\dot{\boldsymbol{M}}_{e}^{*}=\mathbf{C}_{e}^{*}+\boldsymbol{C}_{e}^{* T}$,

$\dot{\boldsymbol{M}}_{b}^{*}=\boldsymbol{C}_{b}^{*}+\boldsymbol{C}_{b}^{* T}$.

In the next section, it will be demonstrated how a coordinated controller can be derived based on the dynamics (22), and its beneficial decoupling properties will be analyzed.

\section{Coordinated control}

The objective of the control is to regulate the pose of the frame $\mathcal{E}$, the position of $\mathcal{C}$, and the orientation of the frame $\mathcal{B}$, around desired values that are fixed in the target frame $\mathcal{T}$. Furthermore, the total angular momentum shall be dumped to stop any inertial drift.

Let us define a set of error coordinates: $\tilde{\boldsymbol{x}}_{c} \in \mathbb{R}^{3}$ is the CoM position error, $\tilde{\boldsymbol{x}}_{e} \in \mathbb{R}^{6}$ is the end-effector pose error, and $\tilde{\boldsymbol{x}}_{b} \in \mathbb{R}^{3}$ the base attitude error. The time-derivatives of the errors can be related to the absolute velocities, as:

$\dot{\tilde{\boldsymbol{x}}}_{c}=\boldsymbol{v}_{c}, \quad \dot{\tilde{\boldsymbol{x}}}_{e}=\boldsymbol{J}_{\tilde{x}_{e}} \boldsymbol{v}_{e}, \quad \dot{\tilde{\boldsymbol{x}}}_{b}=\boldsymbol{J}_{\tilde{x}_{b}} \boldsymbol{\omega}_{b}$,

wherein $\boldsymbol{J}_{\tilde{x}_{e}} \in \mathbb{R}^{6 \times 6}$ and $\boldsymbol{J}_{\tilde{x}_{b}} \in \mathbb{R}^{3 \times 3}$ are the Jacobian matrices of coordinates representation. For orientation, the vector part of the error quaternion is used [36]. The exact expressions of $\tilde{\boldsymbol{x}}_{c}, \tilde{\boldsymbol{x}}_{e}, \tilde{\boldsymbol{x}}_{b}$, $\boldsymbol{J}_{\tilde{x}_{e}}$, and $\boldsymbol{J}_{\tilde{x}_{b}}$ can be found in [30, Sect.IV].

The controller of the external motion is designed as

$\mathcal{F}_{c}=-\boldsymbol{Q}_{c}^{T} \boldsymbol{K}_{c} \tilde{\boldsymbol{x}}_{c}-\boldsymbol{D}_{h} \boldsymbol{h}$,

where $\boldsymbol{K}_{c} \in \mathbb{R}^{3 \times 3}$ is a symmetric, positive definite stiffness matrix, $\boldsymbol{D}_{h} \in \mathbb{R}^{6 \times 6}$ is a positive definite momentum gain matrix. The controller (25a) ensures that the CoM is stabilized around a desired position and that any residual momentum is extracted from the system. The controllers for the end-effector and base tasks are designed as

$$
\begin{aligned}
\breve{\mathcal{F}}_{e}^{i n t} & =-\boldsymbol{J}_{\tilde{x}_{e}}^{T} \boldsymbol{K}_{e} \tilde{\boldsymbol{x}}_{e}-\boldsymbol{D}_{e} \boldsymbol{v}_{e}-\boldsymbol{C}_{b e}^{* T} \breve{\boldsymbol{\omega}}_{b}^{\text {int }}, \\
\breve{\boldsymbol{\tau}}_{b}^{\text {int }} & =-\boldsymbol{J}_{\tilde{x}_{b}}^{T} \boldsymbol{K}_{b} \tilde{\boldsymbol{x}}_{b}-\boldsymbol{D}_{b} \boldsymbol{\omega}_{b},
\end{aligned}
$$

where $\boldsymbol{K}_{e} \in \mathbb{R}^{6 \times 6}$ and $\boldsymbol{K}_{b} \in \mathbb{R}^{3 \times 3}$ are symmetric, positive definite stiffness matrices, and $\boldsymbol{D}_{e} \in \mathbb{R}^{6 \times 6}$ and $\boldsymbol{D}_{b} \in \mathbb{R}^{3 \times 3}$ are positive definite damping matrices. The controllers (25b) and (25c) include a PD-like term, whereas (25b) includes an additional term for compensating the Coriolis and centrifugal coupling of the base into the end-effector. This term is necessary for the proof of stability shown in Section 4.1.

\subsection{Stability analysis}

The closed-loop dynamics resulting from the application of (25) in (22) results in the state-space form (see Appendix A)

$\dot{\chi}=\boldsymbol{f}(\chi), \quad \chi=\left[\begin{array}{c}\boldsymbol{h} \\ \tilde{\boldsymbol{x}}_{c} \\ \boldsymbol{v}_{e}^{\text {int }} \\ \tilde{\boldsymbol{x}}_{e} \\ \breve{\omega}_{b}^{\text {int }} \\ \tilde{\boldsymbol{x}}_{b}\end{array}\right] \in D=\mathbb{R}^{27}$.

The details of (26) are given in (55) in Appendix A. Note that the $27<2(6+n)=30$ because the RW angles are not controlled and are not a state of (3). Let us first define a region that excludes the singularities of $\breve{\boldsymbol{\Gamma}}\left(\boldsymbol{R}_{b}, \boldsymbol{q}_{m}\right)$, as

$\Omega=\left\{\chi \in D: \sigma_{\min }\left(\breve{\boldsymbol{T}}\left(\mathbf{g}\left(\tilde{\boldsymbol{x}}_{c}, \tilde{\boldsymbol{x}}_{e}, \tilde{\boldsymbol{x}}_{b}\right)\right)\right)>0\right\}$,

where the function $\mathbf{g}: \mathbb{R}^{12} \rightarrow \mathbb{R}^{n_{m}}$ is a local one-to-one mapping such that $\boldsymbol{q}_{m}=\mathbf{g}\left(\tilde{\boldsymbol{x}}_{c}, \tilde{\boldsymbol{x}}_{e}, \tilde{\boldsymbol{x}}_{b}\right)$, see Appendix A. The singularities excluded from the region $\Omega$ may in general be a non-zero dimensional subset of $D$.

The proof is based on the application of conditional stability theory [37, p. 45]. The formulation in [38] is used herein, whose main result is reported here for the subcase of stability of equilibrium points:

Theorem 1 (Conditional Stability [38]). Let $\boldsymbol{z}_{0}$ be an equilibrium point of $\dot{\boldsymbol{z}}=\boldsymbol{f}(\boldsymbol{z})$, and let $V(\boldsymbol{z})$ be a $C^{1}$ function such that $V(\boldsymbol{z}) \geq 0$, $V\left(\boldsymbol{z}_{0}\right)=0$ and $\dot{V}(\boldsymbol{z}) \leq 0$. If $\boldsymbol{z}_{0}$ is asymptotically stable conditionally to the largest positively invariant set $\mathcal{A}$ within $\{z: \dot{V}(z)=0\}$, then $\boldsymbol{z}_{0}$ is asymptotically stable.

The proof of stability of (26) is conducted on a hierarchical basis, proving the successive convergence of nested subsets [39] by recursively applying Theorem 1 . The following subsets are defined:

$\mathcal{A}_{1}=\left\{\chi \in \Omega: \boldsymbol{h}=\mathbf{0}, \tilde{\boldsymbol{x}}_{c}=\mathbf{0}\right\}$, 
$\mathcal{A}_{2}=\left\{\chi \in \Omega: \boldsymbol{h}=\mathbf{0}, \tilde{\boldsymbol{x}}_{c}=\mathbf{0}, \boldsymbol{v}_{e}^{\text {int }}=\tilde{\boldsymbol{x}}_{e}=\mathbf{0}\right\}$.

Note that $\mathbf{0} \in \mathcal{A}_{2} \subset \mathcal{A}_{1} \subset \Omega$. The main stability result can be now stated:

Proposition 1. Consider the system (26). Under the Assumptions 1 , 2 , and 3 , the equilibrium point $\chi_{0}=\mathbf{0}$ is asymptotically stable.

Proof. The proof is performed in three steps, proving first the asymptotic stability conditionally to $\mathcal{A}_{2}$, then the asymptotic stability conditionally to $\mathcal{A}_{1}$ and, lastly, the asymptotic stability of the equilibrium point without any restrictions.

Step 1. Asymptotic stability of $\chi_{0}$ conditionally to $\mathcal{A}_{2}$

In $\mathcal{A}_{2}$ the conditions $\boldsymbol{h} \equiv \mathbf{0}$ and $\boldsymbol{v}_{e}^{\text {int }} \equiv \mathbf{0}$ are applied to (55). Let us consider the Lyapunov function candidate

$V_{b}=\frac{1}{2} \breve{\boldsymbol{\omega}}_{b}^{i n t}{ }^{T} \boldsymbol{M}_{b}^{*} \breve{\boldsymbol{\omega}}_{b}^{i n t}+\frac{1}{2} \tilde{\boldsymbol{x}}_{b}^{T} \boldsymbol{K}_{b} \tilde{\boldsymbol{x}}_{b},>0, \forall \chi \in \mathcal{A}_{2}: \chi \neq \chi_{0}$,

which satisfies $V_{b}\left(\chi_{0}\right)=0$. Its time-derivative is

$\dot{V}_{b}=\breve{\boldsymbol{\omega}}_{b}^{\text {int }}{ }^{T} \boldsymbol{M}_{b}^{*} \dot{\boldsymbol{\omega}}_{b}^{\text {int }}+\frac{1}{2} \breve{\boldsymbol{\omega}}_{b}^{\text {int }}{ }^{T} \dot{\boldsymbol{M}}_{b}^{*} \breve{\boldsymbol{\omega}}_{b}^{\text {int }}+\dot{\tilde{\boldsymbol{x}}}_{b}^{T} \boldsymbol{K}_{b} \tilde{\boldsymbol{x}}_{b}=$

$\breve{\boldsymbol{\omega}}_{b}^{i n t}{ }^{\mathrm{T}}\left(-\boldsymbol{C}_{b}^{*} \breve{\boldsymbol{\omega}}_{b}^{i n t}-\boldsymbol{D}_{b} \breve{\boldsymbol{\omega}}_{b}^{i n t}-\boldsymbol{J}_{\tilde{x}_{b}^{T}}^{T} \boldsymbol{K}_{b} \tilde{\boldsymbol{x}}_{b}\right)+\frac{1}{2} \breve{\boldsymbol{\omega}}_{b}^{i n t} \dot{\boldsymbol{M}}_{b}^{*} \breve{\boldsymbol{\omega}}_{b}^{i n t}+$

$\breve{\boldsymbol{\omega}}_{b}^{\text {int }} \boldsymbol{J}_{\tilde{x}_{b}}^{T} \boldsymbol{K}_{b} \tilde{\boldsymbol{x}}_{b}=-\breve{\boldsymbol{\omega}}_{b}^{\text {int }}{ }^{T} \boldsymbol{D}_{b} \breve{\boldsymbol{\omega}}_{b}^{\text {int }}+\frac{1}{2} \breve{\boldsymbol{\omega}}_{b}^{\text {int } \mathrm{T}}\left(\dot{\boldsymbol{M}}_{b}^{*}-2 \boldsymbol{C}_{b}^{*}\right) \breve{\boldsymbol{\omega}}_{b}^{\text {int }}$

$=-\breve{\boldsymbol{\omega}}_{b}^{\text {int }}{ }^{T} \boldsymbol{D}_{b} \breve{\boldsymbol{\omega}}_{b}^{\text {int }} \leq 0$,

$\forall \chi \in \mathcal{A}_{2}$,

wherein (55e), (55f), and (23b) are used. Then, $\dot{V}_{b}$ is negative semi-definite, and the stability conditionally to $\mathcal{A}_{2}$ is proven. To prove convergence, LaSalle's invariance principle is applied. It can be seen in (55e), that $\breve{\omega}_{b}^{\text {int }} \equiv \mathbf{0}$ implies $\tilde{\boldsymbol{x}}_{b} \equiv \mathbf{0}$, so $\chi_{0}$ is asymptotically stable conditionally to $\mathcal{A}_{2}$.

Step 2. Asymptotic stability of $\chi_{0}$ conditionally to $\mathcal{A}_{1}$

In $\mathcal{A}_{1}$ the condition $\boldsymbol{h} \equiv \mathbf{0}$ is applied to (55). Theorem 1 is applied considering $\mathcal{A}=\mathcal{A}_{2}$ and restricting all the statements to the subset $\mathcal{A}_{1}$. Let us then consider the function

$V_{e}=\frac{1}{2} \boldsymbol{v}_{e}^{i n t} \boldsymbol{M}_{e}^{*} \boldsymbol{v}_{e}^{i n t}+\frac{1}{2} \tilde{\boldsymbol{x}}_{e}^{T} \boldsymbol{K}_{e} \tilde{\boldsymbol{x}}_{e} \geq 0, \quad \forall \chi \in \mathcal{A}_{1}$,

which satisfies $V_{e}\left(\chi_{0}\right)=0$. Its time-derivative is

$\dot{V}_{e}=\boldsymbol{v}_{e}^{i n t^{T}} \boldsymbol{M}_{e}^{*} \dot{\boldsymbol{v}}_{e}^{i n t}+\frac{1}{2} \boldsymbol{v}_{e}^{i n t^{T}} \dot{\boldsymbol{M}}_{e}^{*} \boldsymbol{v}_{e}^{i n t}+\dot{\tilde{\boldsymbol{x}}}_{e} \boldsymbol{K}_{e} \boldsymbol{v}_{e}^{i n t}=$

$\boldsymbol{v}_{e}^{i n t}\left(-\boldsymbol{C}_{e}^{*} \boldsymbol{v}_{e}^{i n t}-\boldsymbol{D}_{e} \boldsymbol{v}_{e}^{i n t}-\boldsymbol{J}_{\tilde{x}_{e}}^{T} \boldsymbol{K}_{e} \tilde{\boldsymbol{x}}_{e}\right)+\frac{1}{2} \boldsymbol{v}_{e}^{i n t T} \dot{\boldsymbol{M}}_{e}^{*} \boldsymbol{v}_{e}^{i n t}+$

$\boldsymbol{v}_{e}^{\text {int }} \boldsymbol{J}_{\tilde{x}_{e}}^{T} \boldsymbol{K}_{e} \boldsymbol{v}_{e}^{\text {int }}=-\boldsymbol{v}_{e}^{\text {int }}{ }^{T} \boldsymbol{D}_{e} \boldsymbol{v}_{e}^{\text {int }} \leq 0, \quad \forall \chi \in \mathcal{A}_{1}$,

wherein (55c), (55d), and (23a) are used. Then, $V_{e}$ satisfies the conditions of Theorem 1. By inserting $\boldsymbol{h}=\mathbf{0}$ and $\boldsymbol{v}_{e}^{\text {int }}=\mathbf{0}$ in (55c), it can be seen that $\mathcal{A}_{2}$ is the largest invariant set such that $\dot{V}_{e}(\chi)=0$. Then, the further requirement of asymptotic stability of $\chi_{0}$ conditionally to $\mathcal{A}=\mathcal{A}_{2}$ is guaranteed by Step 1 . By application of Theorem 1 , it follows that $\chi_{0}$ is asymptotically stable conditionally to $\mathcal{A}_{1}$.

Step 3. Asymptotic stability of $\chi_{0}$

The asymptotic stability is proven by application of Theorem 1 , considering $\mathcal{A}=\mathcal{A}_{1}$. Let us consider the function

$V_{h}=\frac{1}{2} \boldsymbol{h}^{T} \boldsymbol{h}+\frac{1}{2} m \tilde{\boldsymbol{x}}_{c}^{T} \boldsymbol{K}_{c} \tilde{\boldsymbol{x}}_{c} \geq 0, \quad \forall \chi \in \Omega$,

which satisfies $V_{h}\left(\chi_{0}\right)=0$. Its time-derivative is

$$
\begin{aligned}
\dot{V}_{h} & =\boldsymbol{h}^{T} \dot{\boldsymbol{h}}+m \dot{\tilde{\boldsymbol{x}}}_{c}^{T} \boldsymbol{K}_{c} \tilde{\boldsymbol{x}}_{c}=\boldsymbol{h}^{T}\left(-\boldsymbol{D}_{h} \boldsymbol{h}-\boldsymbol{Q}_{c}^{T} \boldsymbol{K}_{c} \tilde{\boldsymbol{x}}\right) \\
& +\boldsymbol{h}^{T} \mathbf{Q}_{c}^{T} \boldsymbol{K}_{c} \tilde{\boldsymbol{x}}=-\boldsymbol{h}^{T} \boldsymbol{D}_{h} \boldsymbol{h} \leq 0, \quad \forall \chi \in \Omega,
\end{aligned}
$$

where (55a) and (55b) are applied. Then, $V_{h}$ satisfies the conditions of Theorem 1. By inserting $\boldsymbol{h}=\mathbf{0}$ in (55a), it can be easily seen that $\mathcal{A}_{1}$ is the largest invariant set such that $\dot{V}_{h}=0$. Then, the requirement of asymptotic stability of $\chi_{0}$ conditionally to $\mathcal{A}=\mathcal{A}_{1}$ is guaranteed by Step 2 . By applying Theorem 1 , it follows that $\chi_{0}$ is asymptotically stable.

\subsection{Controller properties}

Writing (20) explicitly and considering $\boldsymbol{a}_{c}=\boldsymbol{M}_{c}^{-1} \mathcal{F}_{c}$, the actuators can be related to the new control inputs, as:

$$
\left[\begin{array}{c}
\mathcal{F}_{b} \\
\boldsymbol{\tau}_{w} \\
\boldsymbol{\tau}_{m}
\end{array}\right]=\left[\begin{array}{ccc}
\boldsymbol{A}_{c b}^{T} & \mathbf{0} & \mathbf{0} \\
\boldsymbol{M}_{b w}^{T} \boldsymbol{M}_{b}^{-1} \boldsymbol{A}_{c b}^{T} & \boldsymbol{T}_{e w}^{T} & \breve{\boldsymbol{T}}_{b w}^{T} \\
\boldsymbol{M}_{b m}^{T} \boldsymbol{M}_{b}^{-1} \boldsymbol{A}_{c b}^{T} & \boldsymbol{T}_{e m}^{T} & \breve{\boldsymbol{T}}_{b m}^{T}
\end{array}\right]\left[\begin{array}{c}
\mathcal{F}_{c} \\
\breve{\mathcal{F}}_{e}^{i n t} \\
\breve{\boldsymbol{\tau}}_{b}^{\text {int }}
\end{array}\right] .
$$

From the zeros in (36), it is possible to see that the allocation of the actuators has a triangular pattern. More specifically, the thrusters wrench $\mathcal{F}_{b}$ is only activated to control the global motion and not to actuate either the end-effector or the base attitude task. One important feature of this triangular structure is that the thrusters are not used to realize the end-effector task but to realize the only tasks that cannot be actuated by any other device: the control of the inertial location of the CoM and the dumping of the angular momentum. This structure is a property of the internal velocities and holds with both sets of external-internal decompositions $\boldsymbol{\Gamma}$ and $\breve{\Gamma}$.

Another important feature can be observed based on the special conserving properties of the momentum. After the momentum transient vanishes, the system converges to a stationary situation in which the CoM remains fixed in the inertial space and the momentum remains zero. Therefore, during the entire time of robot maneuvers that do not involve contact or CoM relocation, it will remain $\tilde{\boldsymbol{x}}_{c}=\mathbf{0}, \boldsymbol{h}=\mathbf{0}$, and in turn $\mathcal{F}_{b}=$ 0. Hence, with the proposed controller, all end-effector or base attitude maneuvers that do not involve contact will require no thrusters and will be entirely accomplished by the coordinated actuation of the arm joints and the reaction wheels. The thrusters will be activated only after contact, and their use will be limited to stopping the inertial drift and restoring the CoM location. The main advantage of the above-mentioned features is that the system consumes ideally zero fuel for contact-free robot and base attitude maneuvering. After contact, it consumes only the amount of fuel needed to dump the momentum and restore the CoM. Furthermore, note that this feature is automatically implemented by a single unified continuous controller, and no switching between controllers is required; this is an advantage compared to a hybrid strategy involving discontinuous switching between a free-floating controller and one implementing a control of the base translation.

\section{Experimental validation}

The proposed control method has been validated on the OnOrbit Servicing Simulator (OOS-Sim) hardware-in-the-loop facility at the German Aerospace Center (DLR). The facility exploits industrial robots to simulate the spacecraft dynamics in a gravityfree environment. A test arm is mounted on a simulator arm in a micro-macro configuration (see Fig. 1). The simulator arm reproduces the dynamics of the spacecraft based on a real-time model integration. The test arm is a torque-controlled KUKA KR4+ lightweight robot $(\approx 17 \mathrm{~kg}$ ) with seven degrees-of-freedom, and the simulator arm is a position-controlled KUKA KR120 industrial robot. Note that with this system the space robot controller can be evaluated taking into account real dynamics, sensor noise, time delay, control discretization, and model uncertainties of the test arm. On the other hand, the spacecraft dynamics is obtained via software simulation of a real-time model. The parameters 
of the spacecraft, as well as the simulated nonidealities of the spacecraft can be adapted depending on the specific scenario. In the present work, a rigid spacecraft has been simulated. The rigid body parameters of the spacecraft were: mass $m^{(0)}=150 \mathrm{~kg}$, inertia $I_{\text {sat }}=\operatorname{diag}(22.8,14.1,18.8) \mathrm{kg} \mathrm{m}^{2}$. Three equal reaction wheels with $4.7 \mathrm{~kg}$ mass and $0.0425 \mathrm{~kg} \mathrm{~m}^{2}$ rotor inertia were simulated, with rotation axes aligned with the principal axes of the spacecraft. The control sampling frequency was $1 \mathrm{kHz}$. The RW limitations were simplistically modeled as a saturation of 0.3 $\mathrm{N} \mathrm{m}$. The thrusters were simplistically modeled as a deadzone of $0.5 \mathrm{~N}$ in translation and $0.5 \mathrm{~N} \mathrm{~m}$ in rotation. No thrusters distribution and time-modulation have been considered in the experiment; the behavior with realistic thrusters was addressed by means of numerical simulations and will be discussed in Section 6. More information regarding the facility can be found in [40].

A representative scenario is considered, in which the endeffector is commanded to reach desired locations in the inertial space, and accidental or voluntary contact happens. The scenario may be divided in the following phases:

- "pre-contact": the end-effector is steered towards the desired locations while no contact force acts on the space robot;

- "contact": a contact force acts on the space robot and transfers momentum to it;

- "post-contact": the contact has terminated and the space robot is subject to the effects of the momentum induced by the contact.

The aim of the following experiments is to validate the controller for the pre-contact and post-contact phases. The analysis of the contact phase would require additional study, and is not treated in the present work. Two sets of experiments were performed:

1. a series of contact-free end-effector maneuvers, to validate the controller in a representative pre-contact situation;

2. a series of experiments in which the end-effector keeps its current position while accumulated momentum is dumped by the thrusters, to validate the behavior in a representative post-contact situation.

In both series of experiments, the state of the base was reconstructed using the simulated model, whereas the end-effector state was reconstructed based on the forward kinematics of the test arm. The CoM and momentum states were reconstructed from the base state and the measurements of the joint angles (see [32]). In a space robot without reaction wheels, the base state can be reconstructed based on IMU, gyro, LIDAR, joint encoders [41]. For a space robot with reaction wheels, the same method could be extended with an additional measurement of the angles of the wheels. For tumbling targets, target motion prediction may be employed in the estimation algorithm [41,42]. The behavior of the robot during both series of experiments can be seen in the accompanying video.

\subsection{Experimental results: pre-contact phase}

A sequence of two end-effector maneuvers was tested. For each maneuver, the end-effector was commanded to reach a desired pose and then to come back. In the first maneuver, a displacement $\Delta \boldsymbol{p}_{e}=[35,0,-25] \mathrm{cm}$ of the position and $\Delta \phi_{e}=[0,-20,0]$ deg of the rotation (roll, pitch, and yaw angles) was commanded to the end-effector. In the second maneuver, an end-effector displacement of $\Delta \boldsymbol{p}_{e}=[23.5,-27,-30] \mathrm{cm}$ and $\Delta \boldsymbol{\phi}_{e}=[-6.7,-6.7,30] \mathrm{deg}$ was commanded. The second maneuver excites three-dimensional effects more pronouncedly. During all maneuvers, the attitude of the spacecraft and the CoM of the whole robot were commanded to hold the initial values. No contact or initial momentum were simulated in this set of experiments. The stiffness gains used were $\boldsymbol{K}_{e}=\operatorname{blkdiag}\left(\boldsymbol{K}_{e, \text { trasl }}, \boldsymbol{K}_{e, \text { rot }}\right)$ for the end-effector, where $\boldsymbol{K}_{e, \text { trasl }}=1000 \boldsymbol{E} \mathrm{N} \mathrm{m}^{-1}, \boldsymbol{K}_{e, \text { rot }}=$

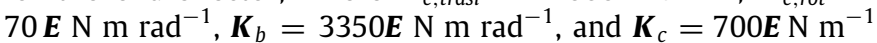
were used for the attitude and the CoM, respectively. The EE and attitude damping gains were designed using the method in [34, p. 36] based on the inertia in the initial configuration. The momentum gain was $\boldsymbol{D}_{h}=\operatorname{blkdiag}\left(\boldsymbol{D}_{p}, \boldsymbol{D}_{l}\right)$ where $\boldsymbol{D}_{p}=3.93 \boldsymbol{E} \mathrm{s}^{-1}$ was used for the linear momentum, and $\boldsymbol{D}_{l}=16 \boldsymbol{E} \mathrm{s}^{-1}$ for the angular momentum.

Fig. 3 shows the results for both maneuvers; the second maneuver starts at $t=t_{2}$. The figure shows the time responses of the end-effector position, the attitude of the spacecraft, the CoM of the whole robot, and the velocity of the reaction wheels, as well as the forces and torques actuated by the thrusters, the reaction wheels', and the joints' drives. It can be observed that the end-effector successfully converged to the desired position after maneuvering. The attitude of the spacecraft was displaced due to the robot motion, but the control action successfully restored it after the maneuver had finished. A longer-lasting oscillation of the attitude of the spacecraft was observed during the lateral maneuver and it was related to the more pronounced excitation of the 1DOF null space of the arm, which was only indirectly damped through the natural friction in the joints. The position of the spacecraft freely moved and was displaced to new locations after each maneuver. On the other hand, the CoM stayed in place and was not affected by the end-effector control. This is a property of the proposed decomposition of the internal and external forces in (36): in a nominal starting condition in which the CoM error and the momentum are zero, the thrusters command $\mathcal{F}_{b}$ does not get activated during end-effector and attitude maneuvering and, consequently, the CoM does not get excited by the coordinated controller. Thanks to this property, the endeffector maneuvers were accomplished entirely by the joints of the arm and the reaction wheels only, as observed in Fig. 3, and exactly zero fuel was consumed. The experiment confirmed the main property of the proposed actuation decomposition, namely, during the pre-contact phase nominally no fuel is consumed by the control.

The steady-state joint torque in Fig. 3 was due to the static friction in the joints of the arm. ${ }^{2}$ Although the maximum robot speed was limited by the low torque capability of the wheels, the RW torque was within the allowable limit of $0.3 \mathrm{Nm}$ for the considered maneuver, proving that the controller is implementable with realistic wheels. Lastly, the RW velocity was low and well below the limits of common devices, e.g. $5000 \mathrm{rpm}$, and returned to zero after each maneuver ended. Based on this, in accordance with the conservation of the zero initial momentum, the actuation can be interpreted as a mutual exchange of momentum between arm and reaction wheels, with the wheels' momentum returning to zero as soon as the arm comes to rest.

\subsection{Experimental results: post-contact phase}

Experiments were conducted to validate the effectiveness of the CoM and momentum dumping tasks in a post-contact situation, i.e., one in which the CoM velocity and the angular momentum are nonzero. A series of impulses along different directions was given manually to the end-effector by using a rod. Note that the contact is given only as a mean to excite the momentum of the system and not to analyze the behavior of the system

\footnotetext{
2 This is explained by the fact that the static friction torques represent a constant disturbance for the PD-like controller (25); at equilibrium, the constant
} disturbance is balanced by nonzero control torques. 

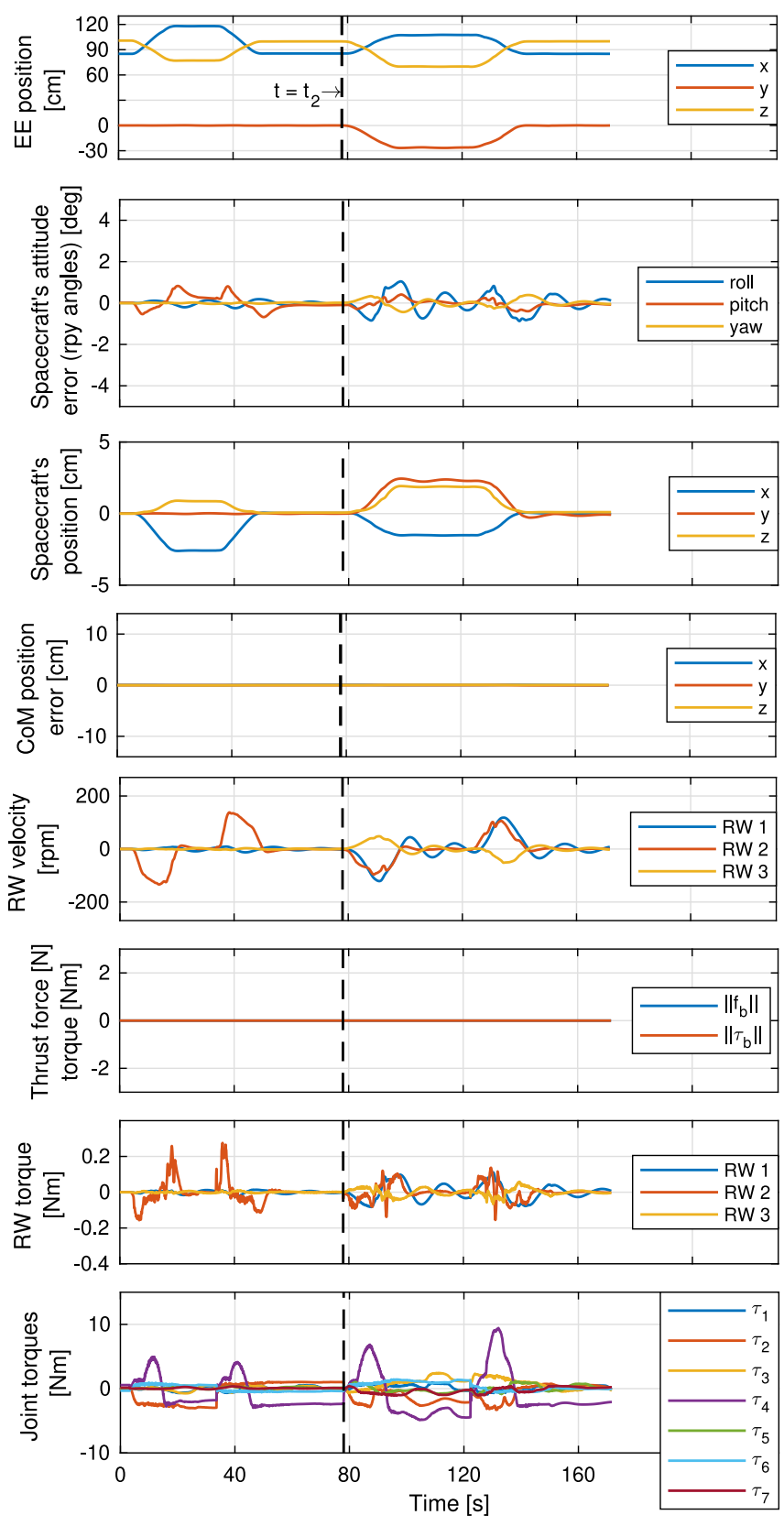

Fig. 3. Response during pre-contact phase.

during contact. The contact wrench needed for the simulation of the spacecraft motion was approximately reconstructed using measurements of the joint torques of the test arm, the details of the reconstruction are omitted for brevity. Note that an accurate reconstruction of the contact wrench is not necessary, being the contact used just as a mean to induce some momentum in the system. For the sake of simplicity, the motion of an eventual target object in the post-contact phase was not considered, and the frame $\mathcal{T}$ was assumed stationary. This assumption holds well for massive target objects and may be removed in future work. In these experiments, the same gains were used except the ones

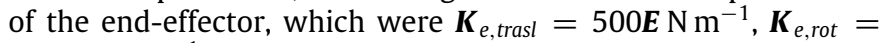
$35 \boldsymbol{E} \mathrm{N} \mathrm{m} \mathrm{rad}^{-1}$.

Fig. 4 shows the reconstructed applied contact force, the CoM position, the angular momentum, and the commanded thrusters forces and torques in the inertial frame. First, observe in Fig. 4 that after each contact the correct CoM location was restored and
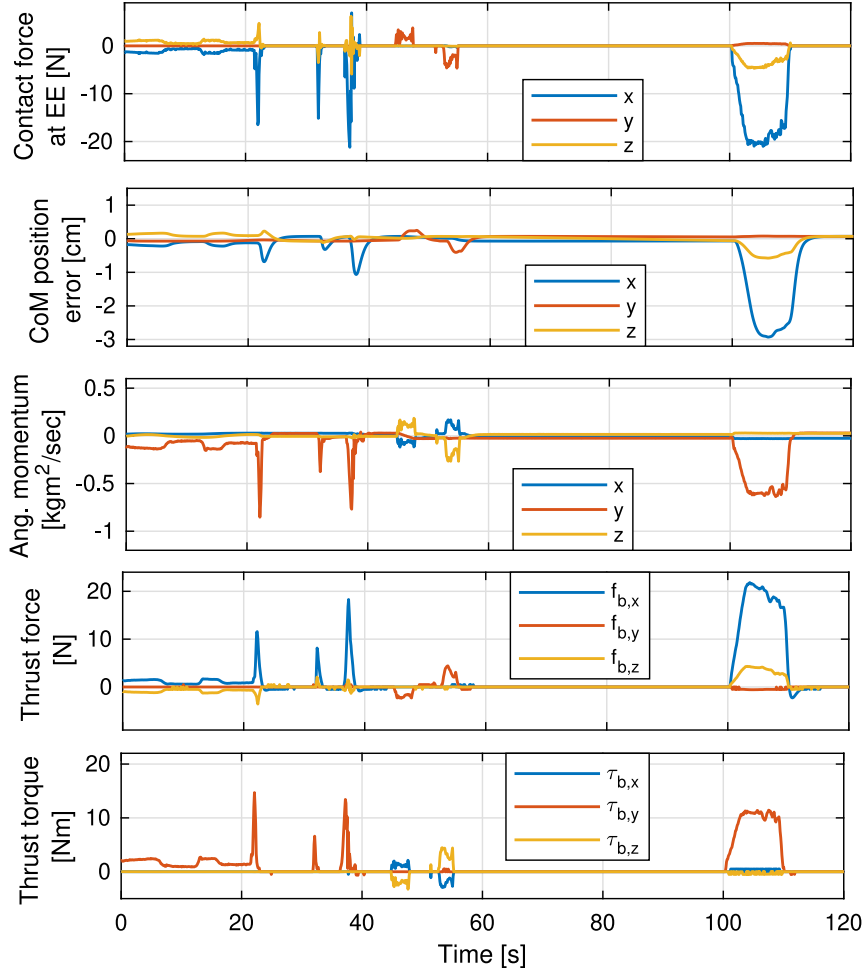

Fig. 4. Response during post-contact phase.

the momentum was dumped. This means that, when the linear and angular momenta got excited, the controller promptly reacted, behaved stably, and accomplished the desired task. Second, observe in Fig. 4 that the impulses were given along different directions and that the controller behaved properly in every direction. The thrusters automatically fired in reaction to the external force. As soon as a CoM displacement was generated along a direction, the thrusters forces reacted in the same direction, but opposite sign.

\subsection{Discussion of the experimental results and limitations}

The series of experiments confirmed that the method is successful in regulating the end-effector pose, the attitude of the spacecraft, and the CoM of the whole robot simultaneously. Furthermore, the experiments confirmed that in the pre-contact phase the control is realized without any use of thrusters, whereas in the post-contact phase the thrusters are automatically activated to restore the proper inertial location of the system and to dump the angular momentum injected by the impulse. This minimalistic use of the thrusters is the main advantage of the proposed method and leads to reduced fuel consumption and improved performance, as preliminary observed in [30], and further analyzed in Section 6.

We point out that a deeper analysis would be needed in order to claim applicability of the same controller also to a contact phase. In particular, the theoretical analysis herein shall be extended to the presence of an applied contact wrench; furthermore, the problem of the impedance matching with a floating target shall be addressed.

In the pre-contact experiments, a longer-lasting oscillation of the attitude was observed during the lateral maneuver and is interpreted as an effect of the arm redundancy. In absence of null-space control, the null-space motion is dissipated entirely by the natural friction in the joints of the robot, which in turn 


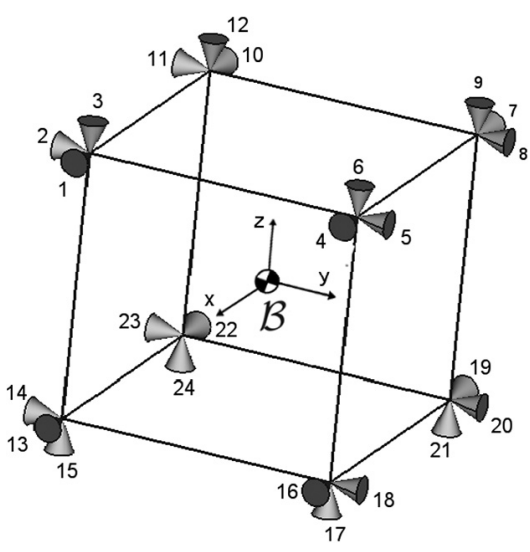

Fig. 5. Thrusters system composed of 24 thrusters arranged on a cubic structure.

reacts on the spacecraft and induces a disturbance on the attitude. The interaction between nullspace, friction, and attitude could be mitigated by implementing a controller of the nullspace, and will be subject of future investigation.

The control method requires that the $\breve{\boldsymbol{T}}\left(\boldsymbol{q}_{m}\right)$ be nonsingular; at singularity, a loss of controllability along a singular direction may happen. The method could be extended with a singularity avoidance [34, p. 38] to limit the occurrence of singularity. In practice, suitably pre-planned trajectories $[18,43]$ are normally employed to limit the occurrence of singularities, and may be employed also in the present case.

\section{Performance with discrete thrusters}

Numerical simulations were carried out to validate the applicability of the controller considering a representative thrusters system. The thrusters system is composed of a redundant set of $n_{t}=24$ thrusters $^{3}$ providing full actuation capability on all translational and rotational components. The thrusters are arranged on a cubic geometry (see Fig. 5), which is a simplified but still meaningful one for the purpose of investigating the control behavior; the optimization of the thrusters geometry for design purposes is not part of the present study. The cube has an edge length of $1 \mathrm{~m}$, and its center is located on the origin of the frame $\mathcal{B}$ (see Fig. 5). On each vertex, three thrusters are mounted, pointing along the $\mathrm{x}, \mathrm{y}, \mathrm{z}$ directions of the frame $\mathcal{B}$.

All thrusters are the same and have the following properties: thrust $u_{\max }=10 \mathrm{~N}$, Minimum Impulse Bit (MIB) $0.15 \mathrm{~N} \mathrm{~s}$, and minimum valve activation time $\Delta t_{\min }=15 \mathrm{~ms}$. The satellite control signal $\mathcal{F}_{b}$ is sampled at a $T_{s a t}=300 \mathrm{~ms}$ rate. On the other hand, the joint and RW control signals, $\boldsymbol{\tau}_{m}$ and $\boldsymbol{\tau}_{w}$ respectively, are sampled at a $T_{\text {joint }}=T_{r w}=1 \mathrm{~ms}$ rate.

\subsection{Thrust distribution}

To resolve the thrusters redundancy, an optimization problem is solved, which takes into account the geometry of the thrusters system, the limitations of the commendable thrust, and a certain optimization criterion. Denoting by $\boldsymbol{u} \in \mathbb{R}^{n_{t}}$ the stacked vector of the desired thrust levels of all thrusters, the satellite control wrench $\mathcal{F}_{b}$ can be related to $\boldsymbol{u}$, as

$$
\mathcal{F}_{b}=\boldsymbol{W u}
$$

\footnotetext{
3 Note that such level of thrusters' redundancy is common in space systems (e.g., see [2]).
}

where $\boldsymbol{W} \in \mathbb{R}^{6 \times n_{t}}$ is the so-called thrust distribution matrix, which is constant and is computed based on the given cubic geometry. No thrusters misalignments are considered in the present work. Eq. (37a) represents an equality constraint for the optimization problem, and is augmented with the following set of inequality constraints that model the thrust as upper-bounded and positive-only:

$0 \leq u_{i} \leq u_{\max }$,

where $u_{i} \in \mathbb{R}$ denotes the $i$ th component of the vector $\boldsymbol{u}$, i.e., the thrust of the $i$ th thruster. The optimization criterion is the minimization of the fuel consumption; at this purpose, the following cost function is considered:

$J=\sum_{i=1}^{n_{t}} u_{i}$.

By adopting (37c), an optimal solution for $\boldsymbol{u}$ is seek at that limits conflicting actuation of the thrusters and thus minimizes the fuel consumption. The selection of the thrusters based on the solution of the linear programming problem (37), is a classic method in the space engineering practice $[44,45]$ and is solved online by application of the simplex algorithm [46] within the $T_{\text {sat }}$ sampling interval.

\subsection{Thruster model and control}

The thrusters are inherently discrete devices that can be operated only in on-off mode. A Pulse-Width-Modulation (PWM) is employed herein to modulate the continuous values $u_{i}$ commanded to the $i$ th thruster to a signal $\bar{u}_{i}$ that can assume either 0 or $u_{\max }$ [47-49].

Given the continuous thrust $u_{i}$ requested over the control interval $T_{\text {sat }}$, a rectangular pulse of amplitude $u_{\max }$ and variable duration is commanded to the thrusters. The pulse is centered at the middle of the control interval and its duration is computed such to yield an equivalent total impulse of the continuous signal, i.e., such that it holds $u_{i} T_{\text {sat }}=u_{\max } \Delta t_{i}$; a deadzone is then set on the signal $\Delta t_{i}$ to model the minimum valve activation time $\Delta t_{\text {min }}$. Based on this, the command to the valve of the $i$ th thruster is modeled in simulation as

$\Delta t_{i}= \begin{cases}\frac{u_{i}}{u_{\max }} T_{\text {sat }} & \frac{u_{i}}{u_{\max }} T_{\text {sat }} \geq \Delta t_{\text {min }} \\ 0 & \frac{u_{i}}{u_{\max }} T_{\text {sat }}<\Delta t_{\text {min }} .\end{cases}$

The $\Delta t_{i}$ signal is discretized with a time step of $T_{p w m}=3 \mathrm{~ms}$.

\subsection{Simulation results}

A unified scenario involving contact as well as contact-free maneuvering was simulated to analyze the effects of the real actuation during a representative capture operation. A sequence of two end-effector maneuvers was first commanded, then a contact was simulated. For each maneuver, the end-effector was commanded to reach a desired pose and then to come back. In both maneuvers, a displacement $\Delta \boldsymbol{p}_{e}=[35 ; 0 ;-25] \mathrm{cm}$ of the position and $\Delta \phi_{e}=[0 ;-20 ; 0]$ deg of the rotation was commanded to the end-effector. The contact consisted in a pure force $\boldsymbol{f}_{e, \text { ext }}=[-10 ; 0 ; 0] \mathrm{N}$ at the end-effector lasting $0.3 \mathrm{~s}$. The simulated robot had the same kinematics and dynamics parameters as those used in the experimental facility. Joint friction with a viscous coefficient of 1.5 was modeled in the simulation. An initial velocity $\boldsymbol{v}_{b}\left(t_{0}\right)=3 \mathrm{~mm} / \mathrm{s}$ of the spacecraft was simulated in order to render the effects of initial nonzero momentum present in practical systems. A disturbance torque $\boldsymbol{\tau}_{b, d}=[5 ; 5 ; 5] \cdot 10^{-4} \mathrm{Nm}$ around the base was simulated to model the effects of environmental forces in orbit. 

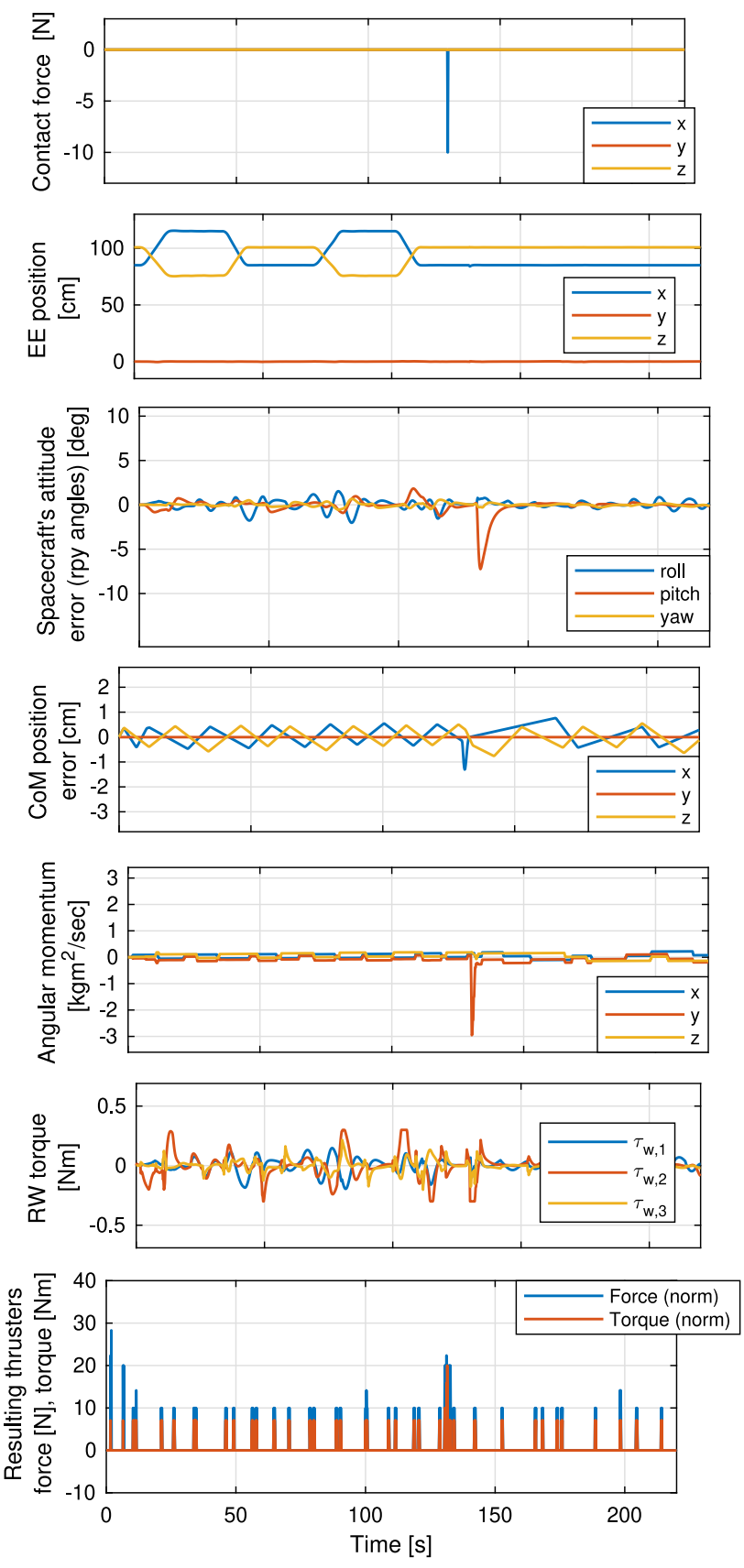

Fig. 6. Performance of the proposed control with discrete thrusters.

One main effect of the thrusters is the necessity of reducing the bandwidth due to discretization. Considering the sampling time $T_{\text {sat }}$ of the thrusters control signal, the bandwidth of the momentum closed-loop system (55a) was decreased to ensure stability; the CoM and momentum gains were decreased such to result in a bandwidth at least 20 times lower than the sampling frequency. The new gains were: $\boldsymbol{K}_{c}=160 \boldsymbol{E} \mathrm{N} \mathrm{m}^{-1}$, $\boldsymbol{D}_{p}=1.878 \boldsymbol{E} \mathrm{s}^{-1}$, and $\boldsymbol{D}_{l}=1 \boldsymbol{E} \mathrm{s}^{-1}$. On the other hand, the endeffector and the attitude gains could be left equal to the ones used in the pre-contact experiment, because with the proposed control the control inputs $\breve{\mathcal{F}}_{e}^{\text {int }}$ and $\breve{\boldsymbol{\tau}}_{b}^{\text {int }}$ are not actuated by the thrusters signal $\mathcal{F}_{b}$, but only by the (fast) torques of the wheels and the arm, e.g. $\boldsymbol{\tau}_{w}$ and $\boldsymbol{\tau}_{m}$, respectively (see (36)).

Fig. 6 shows the induced external force, as well as the time responses of the end-effector position, the spacecraft's attitude error, the CoM position error, and the angular momentum. Furthermore, it shows the control forces acting on the system: the torque commanded to the reaction wheels, and the resulting force $\overline{\boldsymbol{f}}_{b} \in \mathbb{R}^{3}$ and torque $\overline{\boldsymbol{\tau}}_{b} \in \mathbb{R}^{3}$ actuated by the thrusters, given by $\left[\begin{array}{l}\overline{\boldsymbol{f}}_{b} \\ \overline{\boldsymbol{\tau}}_{b}\end{array}\right]=\boldsymbol{W} \overline{\boldsymbol{u}}$, where $\overline{\boldsymbol{u}} \in \mathbb{R}^{n_{t}}$ denotes the stacked vector of the thrusts $\bar{u}_{i}$ of the $i$ th thruster, resulting from the PWM modulation. All vector quantities in the plots are expressed in the inertial frame. Therein it can be observed that the controller worked successfully when considering real thrusters, both during the preand post-contact phases. Namely, the end-effector maneuvers were successfully accomplished, and the spacecraft's attitude was restored after each robot maneuver. After contact, the deviation induced on the attitude was successfully recovered. The CoM error and the angular momentum behaved stably and were kept around zero; however, they reached a limit cycle and did not converge to zero. This is an effect of the minimum valve activation time of the thrusters $\Delta t_{\min }$, which via (38) results in a minimum actuable thrust and thus in a discontinuity of the control signal $\mathcal{F}_{b}$ around zero. This can be observed also in the plot of the reconstructed force and torque in Fig. 6, where it is possible to see that nonzero, albeit sporadic, force and torque were applied in the pre-contact phase. These nonzero force and nonzero torque originate from the nonzero initial momentum modeled in the simulation: at init, the thrusters react as a consequence of the momentum dumping task, then, in turn, the limit cycle is triggered.

The advantages of the proposed control strategy are now analyzed by comparing it with another one which enforces the same requirements but uses only thrusters. More specifically, the proposed controller is compared with the one in [30], which regulates the CoM of the whole system, the attitude of the spacecraft, and the pose of the end-effector, by using only thrusters. The same scenario is considered for both controllers, namely, the same sequence of two end-effector maneuvers is commanded, the same contact is given to the robot, and the same initial conditions are set for the simulation.

One first difference is that the thrusters-only strategy [30] requires reducing the bandwidth of also the attitude system (55a). This is due to the fact that, when using thrusters to actuate the attitude task as in [30], the effect of the low sampling time $T_{\text {sat }}$ plays an important, detrimental role for the tuning of the attitude task. Furthermore, the exact tuning of the bandwidth of the attitude task is not a trivial one, because in [30] the attitude and end-effector task are completely coupled and nonlinear. Herein, maximum attitude gains still yielding a stable behavior were empirically designed, and were $\boldsymbol{K}_{b}=\operatorname{diag}(12,10,10) \mathrm{N} \mathrm{m} \mathrm{rad}^{-1}$. The end-effector and CoM gains were the same used for the proposed control.

Fig. 7 shows the results for the controller [30]. As observed therein, the end-effector maneuver was successfully accomplished, and the system behaved stably after contact. Furthermore, a similar limit cycle was observed on the CoM response, as a consequence of the minimum valve activation. A big displacement of the attitude was however induced during the end-effector maneuver. Interestingly, the attitude deviation in case of thrusters actuation was bigger than the one obtained in case of RW actuation, although the thrusters are capable of providing a higher torque than the RWs. This behavior is interpreted as the joint consequence of the high level of inertial coupling between attitude and end-effector of controller [30], and the limited allowable attitude gains due to the low sampling rate of the thrusters.

Another important difference can be observed in the reconstructed thrusters force and torque in Fig. 7. Therein it is observed how the thrusters were intensively used in the pre-contact phase 

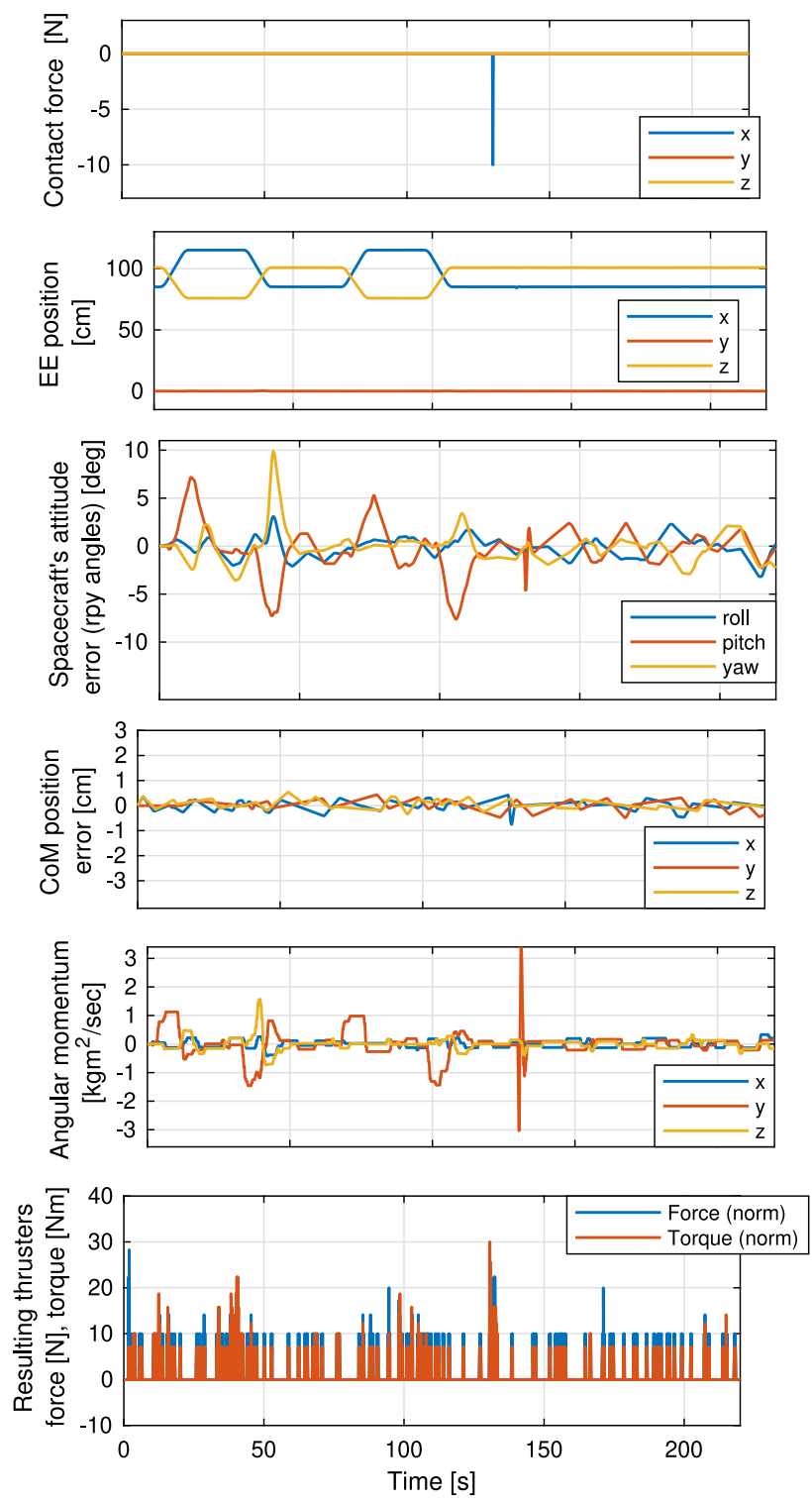

Fig. 7. Performance of the thrusters-only control [30] with discrete thrusters.

in order to counteract the attitude deviation induced by the endeffector maneuver. In contrast, by exploiting the reaction wheels, the proposed controller avoided almost completely any activation of the thrusters during the entire pre-contact phase, saving a considerable amount of fuel. This can be seen also in Fig. 8, which shows a comparison of the active thrusters and the consumed fuel at a given instant for the proposed strategy and the one in [30]. The fuel consumed by the thrusters is computed as [50]

$c=\frac{1}{I_{s p} g_{0}} \int_{0}^{t_{f}} \sum_{i=1}^{n_{t}} \bar{u}_{i} \mathrm{~d} t$,

where $I_{s p}$ is the specific impulse of the thruster and where $g_{0}=9.8 \mathrm{~m} \mathrm{~s}^{-2}$ is the gravitational acceleration at sea level; herein, it is used $I_{s p}=70 \mathrm{~s}$. Observe in Fig. 8 how the thrustersonly strategy required intense thrusters activation during the entire duration of the test sequence, and, therefore, a higher amount of fuel than the combined thrusters and RWs strategy. During the pre-contact maneuvering phase, the thrusters-only control consumed fuel due to the CoM limit cycle, for regulating the attitude, and for cooperating in the actuation of the end-effector task;

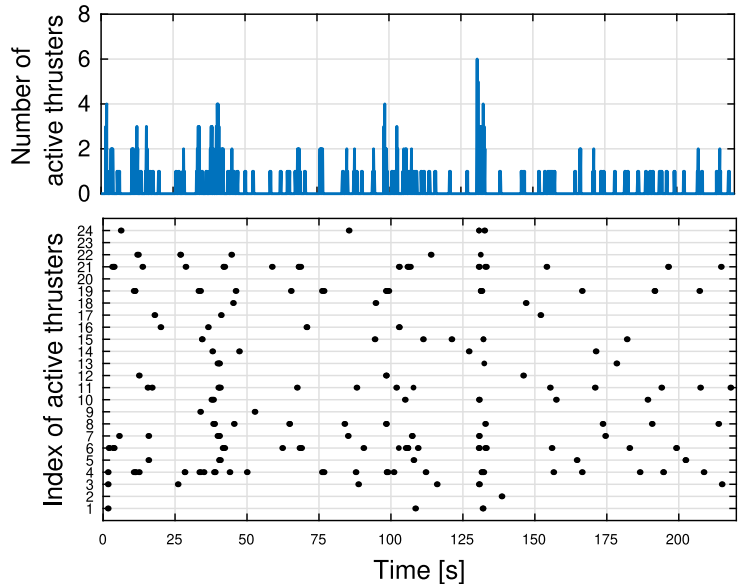

(a) Active thrsuters for thrusters-only control [30]
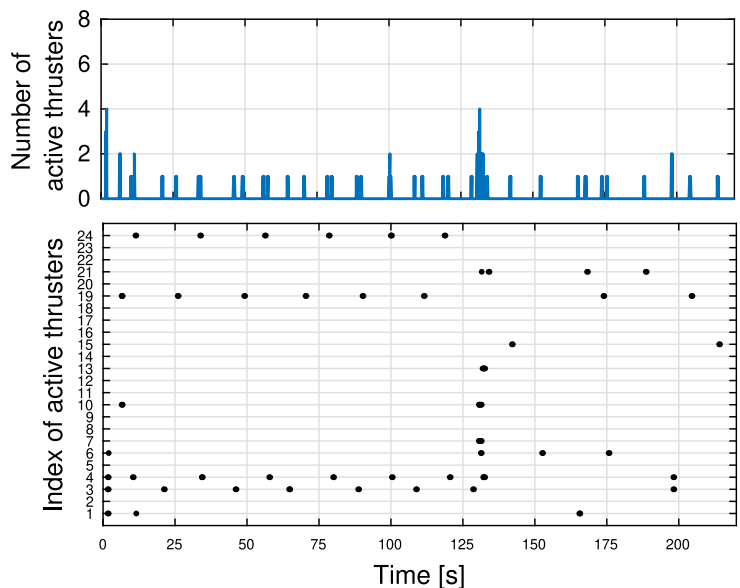

(b) Active thrsuters for proposed control

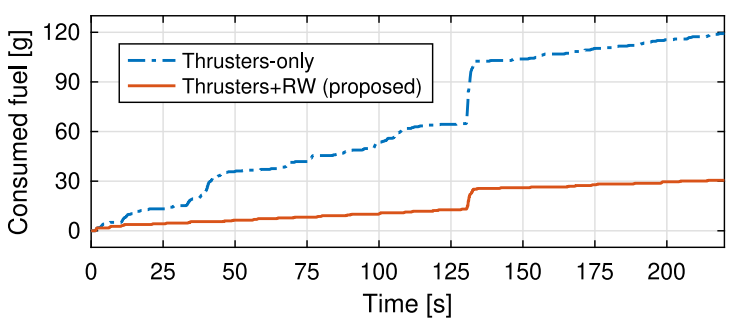

(c) Comparison of fuel

Fig. 8. Comparison of the thrusters usage and the fuel consumption in case of the thrusters-only strategy [30] and the proposed thrusters+RW strategy.

in contrast, the combined thrusters and RWs strategy control consumed fuel only due to the limit cycle. Furthermore, during the contact the RWs strategy exhibited considerably less fuel consumption. Finally, after the contact a comparable amount of fuel was consumed by both strategies due to the limit cycle, and to dump the angular momentum, but the thrusters-only version still exhibited a higher thrusters activity and faster fuel consumption despite no end-effector maneuver had to be counteracted in that phase. In total, for the given test sequence, the thrustersonly control required approximately $290 \%$ more fuel than the proposed combined thruster and RWs method. 


\subsection{Discussion of the simulation results}

The simulation results confirmed that the proposed control works properly when the actuation is accomplished by a representative thrusters system. The main effects of the discrete thrusters were the necessity of suitably reducing the bandwidth of the CoM and angular momentum systems due to the low sampling $T_{s a t}$, and the appearance of a limit cycle due to the minimum valve time $\Delta t_{\min }$. These are typically known effects from satellite control, and are unavoidable considering the abovementioned properties of practical thrusters. The tuning of the magnitude and frequency of the limit cycle is a common problem in the engineering practice [51,52], and could be addressed by varying the parameters $\boldsymbol{K}_{c}, T_{s a t}$, and $T_{p w m}$ based on some tradeoff between accuracy of the CoM position and fuel consumption. The effect of the limit cycle on the proposed control is that during the pre-contact phase the fuel consumption is not exactly zero, as in the case of ideal actuation, but a small amount of fuel is consumed.

The comparison with the controller [30] confirmed the advantage of the proposed control strategy in terms of performance and fuel consumption. The improved performance of the attitude control during end-effector maneuvering is interpreted as being related to the inertial decoupling of the proposed control, whereas in the controller [30] the attitude and end-effector systems are inertially coupled, leading to attitude disturbances that cannot be promptly compensated with the low allowable gains of [30].

The improved fuel consumption of the proposed strategy is related to two main aspects. Firstly, no thrusters effort is wasted to actuate the end-effector task, thanks to the proposed externalinternal decomposition. Secondly, the attitude control task is executed by the reaction wheels, which require only electrical power, and not by the thrusters. These two characteristics altogether lead to a substantial improvement of the fuel consumption.

\section{Conclusions and future work}

A fuel-efficient control method for the coordination of thrusters, reaction wheels, and joints of an orbital robot was derived. A dynamics transformation was introduced, which allowed separating the attitude and end-effector controllers from the thrusters, and achieving the property that ideally zero fuel is consumed in the pre-contact phase. Pre- and post-contact experiments successfully validated the method. Numerical simulations confirmed that the controller may work with realistic thrusters and showed the advantage of the proposed strategy in terms of fuel efficiency and performance. Future work may endow the controller with a null-space control action, and may investigate the applicability of the method to the case of a tumbling target. Furthermore, the extension of the theoretical framework of the proposed method to the contact case will be addressed.

\section{Declaration of competing interest}

The authors declare that they have no known competing financial interests or personal relationships that could have appeared to influence the work reported in this paper.

\section{Appendix A. Dynamic matrices}

The body velocity of the $j$ th body can be expressed as a function of the generalized velocity $v$, as

$$
\boldsymbol{v}_{j}=\underbrace{\left[\begin{array}{lll}
\boldsymbol{A}_{j b}\left(\boldsymbol{q}_{m}\right) & \boldsymbol{J}_{j w} & \boldsymbol{J}_{j m}\left(\boldsymbol{q}_{m}\right)
\end{array}\right]}_{\boldsymbol{J}_{j}\left(\boldsymbol{q}_{m}\right) \in \mathbb{R}^{6 \times(6+n)}} \boldsymbol{v},
$$

where $\boldsymbol{J}_{j w} \in \mathbb{R}^{6 \times n_{w}}$ and $\boldsymbol{J}_{j m}\left(\boldsymbol{q}_{m}\right) \in \mathbb{R}^{6 \times n_{m}}$ are the Jacobian matrices mapping the RW velocities and the velocities of the joints of the arm, respectively, into $\boldsymbol{v}_{j}$. Note that, since the reaction wheels and the arm are on separate kinematics chains, for all bodies of the reaction wheels it holds $\boldsymbol{J}_{j m}\left(\boldsymbol{q}_{m}\right)=\mathbf{0}$, and for all links of the arm it holds $\boldsymbol{J}_{j w}=\mathbf{0}$; additionally, for the reaction wheels $\boldsymbol{A}_{j b}$ is constant because each reaction wheel forms a single-body chain. By convention, the 0th frame is coincident with the base frame $\mathcal{B}$; in turn, it is $\boldsymbol{A}_{0 b}=\boldsymbol{E}, \boldsymbol{J}_{0 w}=\mathbf{0}$, and $\boldsymbol{J}_{0 m}\left(\boldsymbol{q}_{m}\right)=\mathbf{0}$.

The dynamics matrices in (3) are computed as [33]

$$
\begin{aligned}
\boldsymbol{M}\left(\boldsymbol{q}_{m}\right) & =\sum_{j} \boldsymbol{J}_{j}\left(\boldsymbol{q}_{m}\right)^{T} \boldsymbol{\Lambda}_{j}^{(j)} \boldsymbol{J}_{j}\left(\boldsymbol{q}_{m}\right), \\
\boldsymbol{C}\left(\boldsymbol{q}_{m}, \boldsymbol{v}\right) & =\sum_{j} \boldsymbol{J}_{j}\left(\boldsymbol{q}_{m}\right)^{T}\left[\boldsymbol{\Lambda}_{j}^{(j)} \boldsymbol{a}_{j}\left(\boldsymbol{v}_{j}\right)-\boldsymbol{a}_{j}\left(\boldsymbol{v}_{j}\right)^{T} \boldsymbol{\Lambda}_{j}^{(j)}\right] \boldsymbol{J}_{j}\left(\boldsymbol{q}_{m}\right) \\
& +\boldsymbol{J}_{j}\left(\boldsymbol{q}_{m}\right)^{T} \boldsymbol{\Lambda}_{j}^{(j)} \dot{J}_{j}\left(\boldsymbol{q}_{m}\right),
\end{aligned}
$$

where $\Lambda_{j}^{(j)} \in \mathbb{R}^{6 \times 6}$ is the body inertia of the $j$ th and where $\boldsymbol{a}_{j}\left(\boldsymbol{v}_{j}\right) \in \mathbb{R}^{6 \times 6}$ denotes the so-called small adjoint matrix $\boldsymbol{a}_{j}\left(\boldsymbol{v}_{j}\right)=\left[\begin{array}{cc}{\left[\boldsymbol{\omega}_{j}\right]^{\wedge}} & {\left[\boldsymbol{v}_{j}\right]^{\wedge}} \\ \mathbf{0} & {\left[\boldsymbol{\omega}_{j}\right]^{\wedge}}\end{array}\right]$. The sub-blocks of the inertia matrix in (3) are given by

$$
\begin{aligned}
\boldsymbol{M}_{b}\left(\boldsymbol{q}_{m}\right) & =\sum_{j} \boldsymbol{A}_{j b}\left(\boldsymbol{q}_{m}\right)^{T} \boldsymbol{\Lambda}_{j}^{(j)} \boldsymbol{A}_{j b}\left(\boldsymbol{q}_{m}\right) \in \mathbb{R}^{6 \times 6}, \\
\boldsymbol{M}_{b w} & =\sum_{j} \boldsymbol{A}_{j b}\left(\boldsymbol{q}_{m}\right)^{T} \boldsymbol{\Lambda}_{j}^{(j)} \boldsymbol{J}_{j w} \in \mathbb{R}^{6 \times n_{w}}, \\
\boldsymbol{M}_{b m}\left(\boldsymbol{q}_{m}\right) & =\sum_{j} \boldsymbol{A}_{j b}\left(\boldsymbol{q}_{m}\right)^{T} \boldsymbol{\Lambda}_{j}^{(j)} \boldsymbol{J}_{j m}\left(\boldsymbol{q}_{m}\right) \in \mathbb{R}^{6 \times n_{m}}, \\
\boldsymbol{M}_{w} & =\sum_{j} \boldsymbol{J}_{j w}^{T} \boldsymbol{\Lambda}_{j}^{(j)} \boldsymbol{J}_{j w} \in \mathbb{R}^{n_{w} \times n_{w}}, \\
\boldsymbol{M}_{m}\left(\boldsymbol{q}_{m}\right) & =\sum_{j} \boldsymbol{J}_{j m}\left(\boldsymbol{q}_{m}\right)^{T} \boldsymbol{\Lambda}_{j}^{(j)} \boldsymbol{J}_{j m}\left(\boldsymbol{q}_{m}\right) \in \mathbb{R}^{n_{m} \times n_{m}} .
\end{aligned}
$$

\section{Closed-loop equations}

Lemma 1. Given the dynamically consistent nullspace transformation (15), it holds $\boldsymbol{T}_{b} \breve{\boldsymbol{T}}_{b}^{\#}=\boldsymbol{E}$.

Proof. Using (15) and the dynamically consistent pseudoinverse in (17), $\overline{\boldsymbol{T}}_{b}$ can be written as

$\breve{\boldsymbol{T}}_{b}^{\#}=\overline{\boldsymbol{M}}^{-1} \boldsymbol{N}_{e}^{T} \boldsymbol{T}_{b}^{T}\left(\boldsymbol{T}_{b} \boldsymbol{N}_{e} \overline{\boldsymbol{M}}^{-1} \boldsymbol{N}_{e}^{T} \boldsymbol{T}_{b}^{T}\right)^{-1}$.

Then, in order to be $\boldsymbol{T}_{b} \breve{\boldsymbol{T}}_{b}^{\#}=\boldsymbol{E}$, it must be

$\boldsymbol{T}_{b} \overline{\boldsymbol{M}}^{-1} \boldsymbol{N}_{e}^{T} \boldsymbol{T}_{b}^{T}\left(\boldsymbol{T}_{b} \boldsymbol{N}_{e} \overline{\boldsymbol{M}}^{-1} \boldsymbol{N}_{e}^{T} \boldsymbol{T}_{b}^{T}\right)^{-1}=\boldsymbol{E}$,

where (48) was used. By right-multiplying (49) times $\left(\boldsymbol{T}_{b} \boldsymbol{N}_{e} \overline{\boldsymbol{M}}^{-1} \boldsymbol{N}_{e}^{T} \boldsymbol{T}_{b}^{T}\right)$, it is obtained:

$\boldsymbol{T}_{b} \overline{\boldsymbol{M}}^{-1} \boldsymbol{N}_{e}^{T} \boldsymbol{T}_{b}^{T}=\boldsymbol{T}_{b} \boldsymbol{N}_{e} \overline{\boldsymbol{M}}^{-1} \boldsymbol{N}_{e}^{T} \boldsymbol{T}_{b}^{T}$.

Inserting the transpose of (16) into (50), it is

$$
\begin{aligned}
\boldsymbol{T}_{b} \overline{\boldsymbol{M}}^{-1} \boldsymbol{N}_{e}^{T} \boldsymbol{T}_{b}^{T} & =\boldsymbol{T}_{b}\left(\boldsymbol{E}-\boldsymbol{T}_{e}^{\#} \boldsymbol{T}_{e}\right) \overline{\boldsymbol{M}}^{-1} \boldsymbol{N}_{e}^{T} \boldsymbol{T}_{b}^{T}, \\
\mathbf{0} & =-\boldsymbol{T}_{b} \boldsymbol{T}_{e}^{\#} \boldsymbol{T}_{e} \overline{\boldsymbol{M}}^{-1} \boldsymbol{N}_{e}^{T} \boldsymbol{T}_{b}^{T} .
\end{aligned}
$$

Now, given the well-known property $\boldsymbol{T}_{e} \overline{\boldsymbol{M}}^{-1} \boldsymbol{N}_{e}^{T}=\mathbf{0}$ of the dynamically-consistent projector $\boldsymbol{N}_{e}^{T}$ [34, p. 56], it is seen that (52) is always satisfied, proving that $\boldsymbol{T}_{b} \breve{\boldsymbol{T}}_{b}^{\#}=\boldsymbol{E}$. 
To find the closed-loop equations, let us compute the inverse of (18), as [53]

$\dot{\boldsymbol{q}}=\boldsymbol{T}_{e}^{\#} \boldsymbol{v}_{e}^{i n t}+\breve{\boldsymbol{T}}_{b}^{\#} \breve{\boldsymbol{\omega}}_{b}^{i n t}$.

Then, by inserting (53) into (11b), and then applying (10b) and Lemma 1, it is possible to write

$\boldsymbol{\omega}_{b}=\breve{\boldsymbol{\omega}}_{b}^{\text {int }}+\boldsymbol{T}_{b} \boldsymbol{T}_{e}^{\#} \boldsymbol{v}_{e}^{\text {int }}+\boldsymbol{Q}_{b} \boldsymbol{M}_{b}^{-1} \boldsymbol{A}_{c b}^{T} \boldsymbol{h}$.

The closed-loop equations are then found by inserting, (5), (8b), (54), and (25), into (22) and (24), as

$\dot{\boldsymbol{h}}+\boldsymbol{D}_{h} \boldsymbol{h}+\boldsymbol{Q}_{c}^{T} \boldsymbol{K}_{c} \tilde{\boldsymbol{x}}=\mathbf{0}$,

$\dot{\tilde{\boldsymbol{x}}}_{c}=\frac{1}{m} \boldsymbol{Q}_{c} \boldsymbol{h}$,

$\boldsymbol{M}_{e}^{*} \dot{\boldsymbol{v}}_{e}^{i n t}+\boldsymbol{C}_{e}^{*} \boldsymbol{v}_{e}^{i n t}+\boldsymbol{D}_{e} \boldsymbol{v}_{e}^{i n t}+\boldsymbol{J}_{\tilde{x}_{e}}^{T} \boldsymbol{K}_{e} \tilde{\boldsymbol{x}}_{e}$ $=-\left(\boldsymbol{C}_{e h}^{*}+\boldsymbol{D}_{e} \boldsymbol{A}_{e b} \boldsymbol{M}_{b}^{-1} \boldsymbol{A}_{c b}^{T}\right) \boldsymbol{h}$,

$\dot{\tilde{\boldsymbol{x}}}_{e}=\boldsymbol{J}_{\tilde{x}_{e}} \boldsymbol{v}_{e}^{i n t}+\boldsymbol{J}_{\tilde{x}_{e}} \boldsymbol{A}_{e b} \boldsymbol{M}_{b}^{-1} \boldsymbol{A}_{c b}^{T} \boldsymbol{h}$,

$\boldsymbol{M}_{b}^{*} \dot{\boldsymbol{\omega}}_{b}^{i n t}+\boldsymbol{C}_{b}^{*} \breve{\boldsymbol{\omega}}_{b}^{\text {int }}+\boldsymbol{D}_{b} \breve{\boldsymbol{\omega}}_{b}^{\text {int }}+\boldsymbol{J}_{\tilde{x}_{b}}^{T} \boldsymbol{K}_{b} \tilde{\boldsymbol{x}}_{b}$

$=\left(-\boldsymbol{C}_{b e}^{*}-\boldsymbol{D}_{b} \boldsymbol{T}_{b} \boldsymbol{T}_{e}^{\#}\right) \boldsymbol{v}_{e}^{i n t}-\left(\boldsymbol{C}_{b h}^{*}+\boldsymbol{D}_{b} \boldsymbol{Q}_{b} \boldsymbol{M}_{b}^{-1} \boldsymbol{A}_{c b}^{T}\right) \boldsymbol{h}$,

$\dot{\tilde{\boldsymbol{x}}}_{b}=\boldsymbol{J}_{\tilde{x}_{b}} \breve{\boldsymbol{\omega}}_{b}^{i n t}+\boldsymbol{J}_{\tilde{x}_{b}} \boldsymbol{T}_{b} \boldsymbol{T}_{e}^{\#} \boldsymbol{v}_{e}^{i n t}+\boldsymbol{J}_{\tilde{x}_{b}} \boldsymbol{Q}_{b} \boldsymbol{M}_{b}^{-1} \boldsymbol{A}_{c b}^{T} \boldsymbol{h}$.

The matrices $\boldsymbol{M}_{e}^{*}, \boldsymbol{M}_{b}^{*}, \boldsymbol{M}_{b}, \boldsymbol{T}_{b}, \boldsymbol{T}_{e}^{\#}, \breve{\boldsymbol{T}}_{b}^{\#}$, and $\boldsymbol{A}_{e b}$ in (55) are functions of $\boldsymbol{q}_{m}$; the matrix $\boldsymbol{A}_{c b}$ is function of $\boldsymbol{q}_{m}$ and $\tilde{\boldsymbol{x}}_{b}$; the matrices $\boldsymbol{C}_{e}^{*}$ and $\boldsymbol{C}_{b}^{*}$ are functions of $\boldsymbol{q}_{m}, \tilde{\boldsymbol{x}}_{b}, \boldsymbol{v}_{b}, \dot{\boldsymbol{q}}_{w}$, and $\dot{\boldsymbol{q}}_{m}$. The dependency on $\boldsymbol{v}_{b}, \dot{\boldsymbol{q}}_{w}$, and $\dot{\boldsymbol{q}}_{m}$ is replaced with $\boldsymbol{h}, \boldsymbol{v}_{e}^{\text {int }}$, and $\breve{\boldsymbol{\omega}}_{b}^{\text {int }}$ using the inverse of (19). To replace the dependency on $\boldsymbol{q}_{m}$, let us consider the kinematics relation $\tilde{\boldsymbol{x}}_{e}=\boldsymbol{h}\left(\tilde{\boldsymbol{x}}_{c}, \tilde{\boldsymbol{x}}_{b}, \boldsymbol{q}_{m}\right)$. In a local region, the relation is one-to-one and the inverse can be written, as $\boldsymbol{q}_{m}=\mathbf{g}\left(\tilde{\boldsymbol{x}}_{c}, \tilde{\boldsymbol{x}}_{e}, \tilde{\boldsymbol{x}}_{b}\right)$. Then, (55) is an autonomous system with state $\chi=\left[\begin{array}{llllll}\boldsymbol{h}^{T} & \tilde{\boldsymbol{x}}_{c}^{T} & \boldsymbol{v}_{e}^{\text {int }}{ }^{T} & \tilde{\boldsymbol{x}}_{e}^{T} & \breve{\boldsymbol{\omega}}_{b}^{\text {int }}{ }^{T} & \tilde{\boldsymbol{x}}_{b}^{T}\end{array}\right]^{T} \in D$, and domain $D=\left\{\chi \in \mathbb{R}^{27}: \quad \mathbf{g}\left(\tilde{\boldsymbol{x}}_{c}, \tilde{\boldsymbol{x}}_{e}, \tilde{\boldsymbol{x}}_{b}\right)\right.$ is one-to-one $\}$.

\section{Momentum Coriolis-centrifugal terms}

Herein it is shown that the momentum Coriolis-centrifugal vector terms in (21) are zero. Let us preliminary rewrite (4) compactly, as

$\boldsymbol{h}=\boldsymbol{L} \boldsymbol{v}, \quad \boldsymbol{L}=\boldsymbol{A}_{c b}^{-T}\left[\begin{array}{lll}\boldsymbol{M}_{b} & \boldsymbol{M}_{b w} & \boldsymbol{M}_{b m}\end{array}\right]$,

and report the formulas for the transformation of the inertia and Coriolis/centrifugal matrix in (21), as

$$
\begin{aligned}
\breve{\boldsymbol{M}} & =\breve{\boldsymbol{\Gamma}}^{-T} \boldsymbol{M} \breve{\boldsymbol{\Gamma}}^{-1}, \\
\breve{\boldsymbol{C}} & =\breve{\boldsymbol{\Gamma}}^{-T}\left(\boldsymbol{C}-\boldsymbol{M} \breve{\boldsymbol{\Gamma}}^{-1} \dot{\boldsymbol{\Gamma}}\right) \breve{\boldsymbol{\Gamma}}^{-1} .
\end{aligned}
$$

According to the Newton's third law, only external forces do change the total momentum of the system. Therefore, projecting the base external wrench $\mathcal{F}_{b}$ around $\mathcal{C}$, it must be

$\dot{\boldsymbol{h}}=\boldsymbol{A}_{c b}^{-T} \mathcal{F}_{b}$.

Let us now rederive $\boldsymbol{h}$ taking the time derivative of (56), as

$\dot{\boldsymbol{h}}=\dot{\boldsymbol{L}} \boldsymbol{v}+\boldsymbol{L} \dot{\boldsymbol{v}}=\left(\dot{\boldsymbol{L}}-\mathbf{L} \boldsymbol{M}^{-1} \boldsymbol{C}\right) \boldsymbol{v}+\boldsymbol{L} \boldsymbol{M}^{-1} \boldsymbol{f}$,

where (3) is applied. By algebraic manipulation it can be shown that $\boldsymbol{L} \boldsymbol{M}^{-1}=\left[\begin{array}{lll}\boldsymbol{A}_{c b}^{-T} & \mathbf{0} & \mathbf{0}\end{array}\right]$, thus (60) reduces to

$\dot{\boldsymbol{h}}=\left(\dot{\boldsymbol{L}}-\boldsymbol{L} \boldsymbol{M}^{-1} \boldsymbol{C}\right) \boldsymbol{v}+\boldsymbol{A}_{c b}^{-T} \mathcal{F}_{b}$.

Comparing (59) and (61) for all $\boldsymbol{v} \in \mathbb{R}^{n}$ and $\mathcal{F}_{b} \in \mathbb{R}^{6}$, a constraint for the motion is then obtained as [54]

$\left(\dot{\boldsymbol{L}}-\mathbf{L} \boldsymbol{M}^{-1} \boldsymbol{C}\right) \boldsymbol{v}=\mathbf{0}$.
Thanks to the constraint (62) it is then shown that the Coriolis/centrifugal vector terms are identically zero for the momentum equation in (21). In fact, it holds

$$
\begin{aligned}
\breve{\boldsymbol{C}}\left[\begin{array}{c}
\boldsymbol{h} \\
\boldsymbol{v}_{e}^{\text {int }} \\
\breve{\boldsymbol{\omega}}_{b}^{\text {int }}
\end{array}\right] & =\breve{\boldsymbol{\Gamma}}^{-T}\left(\boldsymbol{C}-\boldsymbol{M} \breve{\boldsymbol{\Gamma}}^{-1} \dot{\dot{\boldsymbol{\Gamma}}}\right) \breve{\boldsymbol{\Gamma}}^{-1}\left[\begin{array}{c}
\boldsymbol{h} \\
\boldsymbol{v}_{e}^{\text {int }} \\
\breve{\boldsymbol{\omega}}_{b}^{\text {int }}
\end{array}\right] \\
& =\breve{\boldsymbol{\Gamma}}^{-T} \boldsymbol{M} \breve{\boldsymbol{\Gamma}}^{-1}\left(\breve{\boldsymbol{\Gamma}} \boldsymbol{M}^{-1} \boldsymbol{C}-\dot{\dot{\boldsymbol{\Gamma}}}\right) \boldsymbol{v} \\
& =\breve{\boldsymbol{M}}\left(\breve{\boldsymbol{\Gamma}} \boldsymbol{M}^{-1} \boldsymbol{C}-\dot{\boldsymbol{\Gamma}}\right) \boldsymbol{v} \\
& =-\boldsymbol{M}_{c}^{-1}\left(\dot{\boldsymbol{L}}-\boldsymbol{L} \boldsymbol{M}^{-1} \boldsymbol{C}\right) \boldsymbol{v} \\
& =\mathbf{0},
\end{aligned}
$$

where (19), the block-diagonal inertia in (21), and the momentum constraint (62), are applied.

\section{Appendix B. Supplementary data}

Supplementary material related to this article can be found online at https://doi.org/10.1016/j.robot.2020.103564.

\section{References}

[1] B. Siciliano, O. Khatib, Springer Handbook of Robotics, second ed., Springer Publishing Company, Incorporated, 2016

[2] P. Colmenarejo, J. Branco, N. Santos, P. Serra, J. Telaar, H. Strauch, A.M. Giordano, M.D. Stefano, C. Ott, M. Rainer, D. Henry, J. Jaworski, E. Papadopoulos, G. Visentin, F. Ankersen, J. Gil-Fernandez, Methods and outcomes of the comrade project - design of robust combined control for robotic spacecraft and manipulator in servicing missions, in: 69th International Astronautical Congress, 2018.

[3] A. Flores-Abad, O. Ma, K. Pham, S. Ulrich, A review of space robotics technologies for on-orbit servicing, Prog. Aerosp. Sci. 68 (2014) 1-26, http://dx.doi.org/10.1016/j.paerosci.2014.03.002.

[4] N. Inaba, M. Oda, Autonomous satellite capture by a space robot: world first on-orbit experiment on a Japanese robot satellite ets-vii, in: Proceedings 2000 ICRA. Millennium Conference. IEEE International Conference on Robotics and Automation. Symposia Proceedings (Cat. No.00CH37065), Vol. 2, 2000, pp. 1169-1174, http://dx.doi.org/10.1109/ROBOT.2000.844757.

[5] K. Yoshida, Engineering test satellite vii flight experiments for space robot dynamics and control: Theories on laboratory test beds ten years ago, now in orbit, Int. J. Robot. Res. 22 (5) (2003) 321-335, http://dx.doi.org/10.1177/0278364903022005003, arXiv:https://doi.org/10. $1177 / 0278364903022005003$.

[6] Y. Umetani, K. Yoshida, Continuous path control of space manipulators mounted on omv, Acta Astronaut. (ISSN: 0094-5765) 15 (12) (1987) 981-986, http://dx.doi.org/10.1016/0094-5765(87)90022-1.

[7] Y. Umetani, K. Yoshida, Resolved motion rate control of space manipulators with generalized jacobian matrix, IEEE Trans. Robot. Autom. 5 (3) (1989) 303-314, http://dx.doi.org/10.1109/70.34766.

[8] Y. Masutani, F. Miyazaki, S. Arimoto, Sensory feedback control for space manipulators, in: IEEE International Conference on Robotics and Automation, Vol. 3, 1989, pp. 1346-1351, http://dx.doi.org/10.1109/ROBOT.1989. 100167.

[9] Z. Vafa, S. Dubowsky, The kinematics and dynamics of space manipulators: The virtual manipulator approach, Int. J. Robot. Res. 9 (4) (1990) 3-21.

[10] E. Papadopoulos, S. Dubowsky, On the nature of control algorithms for free-floating space manipulators, IEEE Trans. Robot. Autom. 7 (6) (1991) 750-758, http://dx.doi.org/10.1109/70.105384.

[11] D. Nenchev, K. Yoshida, Y. Umetani, Analysis, design and control of freeflying space robots using fixed-attitude-restricted jacobian matrix, in: Robotics Res.-5th Int. Symp., MIT Press, 1991, pp. 251-258.

[12] D. Nenchev, Y. Umetani, K. Yoshida, Analysis of a redundant free-flying spacecraft/manipulator system, IEEE Trans. Robot. Autom. 8 (1) (1992) 1-6, http://dx.doi.org/10.1109/70.127234.

[13] F. Caccavale, B. Siciliano, Kinematic control of redundant free-floating robotic systems, Adv. Robot. 15 (4) (2001) 429-448, http://dx.doi.org/10. 1163/156855301750398347. 
[14] L. Sentis, O. Khatib, Control of free-floating humanoid robots through task prioritization, in: Proceedings of the 2005 IEEE International Conference on Robotics and Automation, 2005, http://dx.doi.org/10.1109/ROBOT.2005. 1570361.

[15] A.M. Giordano, G. Garofalo, M. De Stefano, C. Ott, A. Albu-Schäffer, Dynamics and control of a free-floating space robot in presence of nonzero linear and angular momenta, in: Proceedings of the 55th IEEE Conference on Decision and Control, 2016.

[16] S. Dubowsky, M.A. Torres, Path planning for space manipulators to minimize spacecraft attitude disturbances, in: Proceedings of the 1991 IEEE International Conference on Robotics and Automation, Vol. 3, 1991, pp. 2522-2528, http://dx.doi.org/10.1109/ROBOT.1991.132005.

[17] K. Yamada, S. Yoshikawa, Y. Fujita, Arm path planning of a space robot with angular momentum, Adv. Robot. 9 (6) (1994) 693-709, http://dx.doi. org/10.1163/156855395X00364.

[18] R. Lampariello, G. Hirzinger, Generating feasible trajectories for autonomous on-orbit grasping of spinning debris in a useful time, in: 2013 IEEE/RSJ International Conference on Intelligent Robots and Systems, 2013, pp. 5652-5659.

[19] H. Nakanishi, K. Yoshida, Impedance control for free-flying space robots -basic equations and applications-, in: 2006 IEEE/RSJ International Conference on Intelligent Robots and Systems, 2006, http://dx.doi.org/10.1109/ IROS.2006.282334.

[20] S. Abiko, R. Lampariello, G. Hirzinger, Impedance control for a free-floating robot in the grasping of a tumbling target with parameter uncertainty, in: 2006 IEEE/RSJ International Conference on Intelligent Robots and Systems, 2006, pp. 1020-1025, http://dx.doi.org/10.1109/IROS.2006.281785.

[21] K. Yoshida, N. Sashida, R. Kurazume, Y. Umetani, Modeling of collision dynamics for space free-floating links with extended generalized inertia tensor, in: IEEE International Conference on Robotics and Automation, Vol. 1, 1992, pp. 899-904, http://dx.doi.org/10.1109/ROBOT.1992.220182.

[22] Liang-Boon Wee, M.W. Walker, On the dynamics of contact between space robots and configuration control for impact minimization, IEEE Trans. Robot. Autom. 9 (5) (1993) 581-591, http://dx.doi.org/10.1109/70.258051.

[23] K. Yoshida, H. Nakanishi, Impedance matching in capturing a satellite by a space robot, in: Proceedings 2003 IEEE/RSJ International Conference on Intelligent Robots and Systems (IROS 2003) (Cat. No.03CH37453), Vol. 4, Vol. 3, 2003, pp. 3059-3064, http://dx.doi.org/10.1109/IROS.2003.1249626.

[24] A.M. Giordano, G. Garofalo, A. Albu-Schäffer, Momentum dumping for space robots, in: 2017 IEEE 56th Annual Conference on Decision and Control (CDC), 2017, pp. 5243-5248, http://dx.doi.org/10.1109/CDC.2017. 8264434.

[25] E. Papadopoulos, S. Dubowsky, Coordinated manipulator/spacecraft motion control for space robotic systems, in: Proceedings. 1991 IEEE International Conference on Robotics and Automation, Vol. 2, 1991, pp. 1696-1701, http://dx.doi.org/10.1109/ROBOT.1991.131864.

[26] S. Dubowsky, E. Papadopoulos, The kinematics, dynamics, and control of free-flying and free-floating space robotic systems, IEEE Trans. Robot. Autom. 9 (5) (1993) 531-543, http://dx.doi.org/10.1109/70.258046.

[27] O. Egeland, J.R. Sagli, Coordination of motion in a spacecraft/ manipulator system, Int. J. Robot. Res. 12 (4) (1993) 366-379, http://dx.doi.org/10.1177/ 027836499301200404.

[28] S.A.A. Moosavian, E. Papadopoulos, On the control of space free-flyers using multiple impedance control, in: Proceedings of International Conference on Robotics and Automation, Vol. 1, 1997, pp. 853-858, http://dx.doi.org/10. 1109/ROBOT.1997.620141.

[29] M. De Stefano, R. Balachandran, A.M. Giordano, C. Ott, C. Secchi, An energybased approach for the multi-rate control of a manipulator on an actuated base, in: 2018 IEEE International Conference on Robotics and Automation (ICRA), 2018, pp. 1072-1077, http://dx.doi.org/10.1109/ICRA.2018.8460497.

[30] A.M. Giordano, C. Ott, A. Albu-Schäffer, Coordinated control of spacecraft's attitude and end-effector for space robots, IEEE Robot. Autom. Lett. (ISSN: 2377-3766) 4 (2) (2019) 2108-2115, http://dx.doi.org/10.1109/LRA.2019. 2899433.

[31] A. Antonello, A. Valverde, P. Tsiotras, Dynamics and control of spacecraft manipulators with thrusters and momentum exchange devices, J. Guid. Control Dyn. 42 (1) (2019) 15-29, http://dx.doi.org/10.2514/1.G003601, arXiv:https://doi.org/10.2514/1.G003601.

[32] A.M. Giordano, D. Calzolari, A. Albu-Schäffer, Workspace fixation for freefloating space robot operations, in: 2018 IEEE International Conference on Robotics and Automation (ICRA), 2018.
[33] G. Garofalo, C. Ott, A. Albu-Schäffer, On the closed form computation of the dynamic matrices and their differentiations, in: 2013 IEEE/RSJ International Conference on Intelligent Robots and Systems, 2013, http://dx.doi.org/10. 1109/IROS.2013.6696688, 2364-2359.

[34] C. Ott, Cartesian Impedance Control of Redundant and Flexible-Joint Robots, Springer, 2008.

[35] O. Khatib, A unified approach for motion and force control of robot manipulators: the operational space formulation, IEEE J. Robot. Autom. 3 (1) (1987) 43-53, http://dx.doi.org/10.1109/JRA.1987.1087068.

[36] C. Natale, Interaction Control of Robot Manipulators: Six Degrees-OfFreedom Tasks, Vol. 3, Springer, Heidelberg, 2003.

[37] P.K.R. Sepulchre, M. Jankovic, Constructive Nonlinear Control, first ed., Springer, 1997

[38] G. Garofalo, C. Ott, Energy based limit cycle control of elastically actuated robots, IEEE Trans. Automat. Control 62 (5) (2017) 2490-2497, http://dx. doi.org/10.1109/TAC.2016.2599781.

[39] A. Dietrich, C. Ott, A. Albu-Schäffer, Multi-objective compliance control of redundant manipulators: hierarchy, control, and stability, in: 2013 IEEE/RS. International Conference on Intelligent Robots and Systems, 2013, pp. 3043-3050.

[40] J. Artigas, M.D. Stefano, W. Rackl, R. Lampariello, B. Brunner, W. Bertleff, R Burger, O. Porges, A. Giordano, C. Borst, A. Albu-Schäffer, The OOS-SIM: An on-ground simulation facility for on-orbit servicing robotic operations, in: 2015 IEEE International Conference on Robotics and Automation (ICRA) 2015, http://dx.doi.org/10.1109/ICRA.2015.7139588.

[41] A. Gallardo, H. Mishra, A.M. Giordano, R. Lampariello, Robust estimation of motion states for free-floating tumbling target capture, in: 2019 IEEE Aerospace Conference, 2019.

[42] H. Mishra, M. De Stefano, A.M. Giordano, C. Ott, A nonlinear observer for free-floating target motion using only pose measurements, in: 2019 American Control Conference (ACC), 2019, pp. 1114-1121.

[43] D. Calzolari, R. Lampariello, A.M. Giordano, Singularity maps of space robots and their application to gradient-based trajectory planning, in: Robotics: Science and Systems (Accepted), 2020.

[44] B. Crawford, Configuration design and efficient operation of redundant multi-jet systems, in: Astrodynamics Conference, arXiv:https://arc.aiaa. org/doi/pdf/10.2514/6.1969-845. URL https://arc.aiaa.org/doi/abs/10.2514/ 6.1969-845, http://dx.doi.org/10.2514/6.1969-845.

[45] E.V. Bergmann, S. Croopnick, J. Turkovich, C. Work, An advanced spacecraft autopilot concept, J. Guid. Control 2 (3) (1979) 161-168, http://dx.doi.org/ 10.2514/3.55855, arXiv:https://doi.org/10.2514/3.55855.

[46] G. Dantzig, Linear programming and extensions, in: Rand Corporation Research Study, Princeton Univ. Press, 1963.

[47] F. Bernelli-Zazzera, P. Mantegazza, Pulse-width equivalent to pulseamplitude discrete control of linearsystems, J. Guid. Control Dyn. 15 (2) (1992) 461-467, http://dx.doi.org/10.2514/3.20858, arXiv:https://doi.org/ $10.2514 / 3.20858$.

[48] L. Shieh, W. Wang, J.W. Sunkel, Design of pam and pwm controllers for sampled-data interval systems, J. Dyn. Syst. Meas. Control 118 (4) (1996) 673-682.

[49] T. Ieko, Y. Ochi, K. Kanai, New design method for pulse-width modulation control systems via digital redesign, J. Guid. Control Dyn. 22 (1) (1999) 123-128, http://dx.doi.org/10.2514/2.4358, arXiv:https://doi.org/10.2514/2. 4358.

[50] O.B. George P. Sutton, Rocket Propulsion Elements, ninth ed., Wiley, 2016.

[51] M.J. Sidi, Spacecraft Dynamics and Control: a Practical Engineering Approach, Vol. 7, Cambridge university press, 1997.

[52] T.D. Krovel, Optimal Tuning of PWPF Modulator for Attitude Control (Master's thesis), Norwegian University of Science and Technology, 2005.

[53] A. Dietrich, C. Ott, J. Park, The hierarchical operational space formulation: Stability analysis for the regulation case, IEEE Robot. Autom. Lett. 3 (2) (2018) 1120-1127, http://dx.doi.org/10.1109/LRA.2018.2792154.

[54] G. Garofalo, B. Henze, J. Englsberger, C. Ott, On the inertially decoupled structure of the floating base robot dynamics, 48 (1) 322-327, http://dx.doi.org/10.1016/j.ifacol.2015.05.189, 8th Vienna International Conferenceon Mathematical Modelling MATHMOD 2015. 


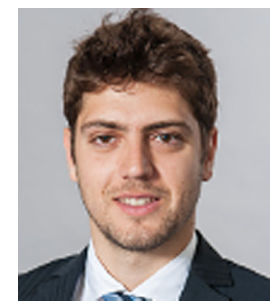

Alessandro M. Giordano is a Research Associate at the Department of Informatics of the Technical University of Munich, in cooperation with the Institute of Robotics and Mechatronics of the German Space Center (DLR). He received his B.Sc. degree in Aerospace Engineering from Università degli studi di Palermo in 2011 and his M.Sc. degree in Astronautical Engineering from Sapienza Università di Roma in 2014. His current research interests include space robotics, nonlinear dynamics and control, and flexible multibody systems.

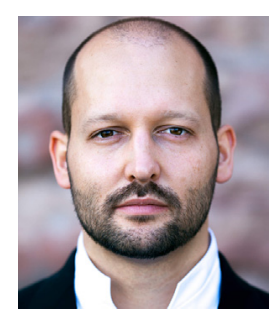

Alexander Dietrich received his Dipl.-Ing. degree in mechanical engineering from the Technical University of Munich (TUM) in 2008 and the Dr.-Ing. degree in 2015. In 2010, he joined the German Aerospace Center (DLR), Institute of Robotics and Mechatronics, as a research scientist. In 2016, he received the Georges Giralt PhD Award for the best European thesis in robotics. He published more than 40 peer-reviewed papers in international journals, books and on conferences. He is Editor for the IEEE ICRA 2020-2022, Associate Editor for the IEEE Robotics and Automation Magazine (RAM), the IEEE Robotics and Automation Letters (RA-L), the IEEE ICRA 2016-2018, and he is co-chair of the IEEE RAS Technical Committee on Whole-Body Control. Since 2017 he is head of the whole-body control group. His current research interests include whole-body mobile manipulation, impedance and force control, hierarchical control for kinematically redundant robots, safe physical human-robot interaction, and the stability analysis of the corresponding control approaches.

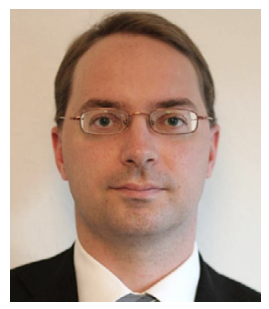

Christian Ott received his doctoral degree in Automatic Control from the University of Saarland, Germany, in 2005. He was a visiting researcher at the University of Twente and worked as a project assistant professor at the University of Tokyo. Currently he is the head of the department for Analysis and Control of Advanced Robotic Systems in the Institute of Robotics and Mechatronics at the German Aerospace Center (DLR). His main research interests are the application of nonlinear control methods to robotic systems, force and impedance control, and control of bipedal humanoid

robots.

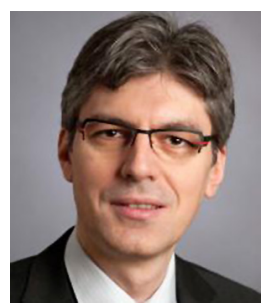

Alin Albu-Schäffer graduated in electrical engineering at the Technical University of Timisoara in 1993 and got the Ph.D. in automatic control from the Technical University of Munich (TUM) in 2002. Since 2012 he is the head of the Institute of Robotics and Mechatronics at the German Aerospace Center (DLR), which he joined in 1995 as a Ph.D. candidate. Moreover, he is a profess or at TUM, holding the Chair for "Sensor-Based Robotic Systems and Intelligent Assistance Systems" at the Computer Science Department. His personal research interests include robot design, modeling and control, flexible joint and variable compliance robots for interaction, and bio-inspired manipulation and locomotion, physical human-robot robot design. 